\title{
Bayesian model selection and parameter estimation for fatigue damage progression models in composites
}

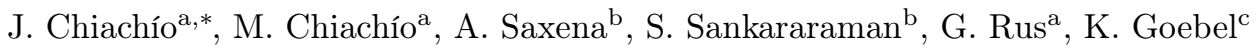 \\ ${ }^{a}$ Dept. Structural Mechanics and Hydraulic Engineering, University of Granada. \\ Campus de Fuentenueva s/n, 18071 Granada, Spain. \\ ${ }^{b}$ SGT Inc., NASA Ames Research Center, Moffett Field, CA 94035-1000 \\ ${ }^{c}$ NASA Ames Research Center, Intelligent Systems Division. Moffett Field, CA 94035-1000
}

\begin{abstract}
A Bayesian approach is presented for selecting the most probable model class among a set of damage mechanics models for fatigue damage progression in composites. Candidate models, that are first parameterized through a Global Sensitivity Analysis, are ranked based on estimated probabilities that measure the extent of agreement of their predictions with observed data. A case study is presented using multi-scale fatigue damage data from a cross-ply carbon-epoxy laminate. The results show that, for this case, the most probable model class among the competing candidates is the one that involves the simplest damage mechanics. The principle of Ockham's razor seems to hold true for the composite materials investigated here since the data-fit of more complex models is penalized, as they extract more information from the data.
\end{abstract}

Keywords: Composites, Fatigue, Damage mechanics, Bayesian methods

\section{Introduction}

Modeling the progression of fatigue damage in fiber-reinforced polymer (FRP) composite materials is still a challenging problem with important implications for safety and cost in a wide range of engineering applications. Although composites are high-performance materials with high strength-weight ratios, they are susceptible to fatigue degradation from the beginning of lifespan [1]. Unlike metals, fatigue in composites is governed by complex multi-scale damage processes driven by several internal fracture events that ultimately lead to the alteration of the macro-scale mechanical properties $[1,2]$. The inherent complexity of this process implies uncertainty in modeling, that not only includes the uncertainty in model parameters but also the uncertainty arising from the choice of a particular model class (e.g., the parameterized mathematical structure of the model for predicting damage behavior). This paper focuses on quantifying the model uncertainty of a set of candidate damage mechanics models for composites, through a full Bayesian approach

\footnotetext{
*Corresponding author. e-mail: jchiachio@ugr.es Tel:(+34)958240037 Fax:(+34)958249959

E.T.S. de Ingenieros de Caminos, Canales y Puertos. 
that simultaneously estimates the plausibility of each individual model class along with the uncertainty of the underlying model parameters.

Some researchers have started investigating the role of uncertainties in modeling the behavior of composites materials. For example, Sriramula and Chryssanthopoulos [3] discussed different stochastic modeling approaches for analyzing uncertainties at the ply-level, coupon-level and component-level. Uncertainty quantification methods have also been used to assess the uncertainty in the material properties $[4,5]$ and extended to study a variety of phenomena such as elastic response [6, 7], aeroelastic behavior [8, 9], and failure [10, 11], among others. While the topic of uncertainty quantification is receiving increased attention in the composites literature, there is still an evident need for a rigorous treatment of the uncertainty in modeling the progression of fatigue damage of composite materials.

In particular, the Bayesian approach has been successfully applied for uncertainty quantification in fatigue, but mainly in the context of metals [12-19]. For example, Cross et al. [13] and Sankararaman et al. [14] used Bayesian inference to estimate parameters underlying crack growth behavior. Sankararaman et al. [16] used dynamic Bayesian networks for model parameter estimation and calculated Bayes factors to quantify model uncertainty. Recently, Chiachío et al. [20] have proposed a stochastic model for damage evolution and used a Bayesian model selection framework to account for model uncertainty. Therefore it seems reasonable to explore the applicability of these methods to fatigue damage modeling in composites materials, where the benefits of the Bayesian approach can be fully exploited due to the inherent complexity and the existence of multiple competing models.

This paper proposes a rigorous Bayesian framework to account for modeling uncertainty in application to the problem of fatigue damage progression in composite materials. To this end, Bayes' Theorem is applied at two levels: first, to quantify the uncertainty regarding the model parameters for a specific model class, and second, to assess the probability of each model class within a set of candidate damage mechanics models. Here, probability is interpreted as a multi-valued logic that expresses the degree of belief of a proposition conditioned on the given information $[21,22]$. Consequently, the approach has the advantage of being able to quantify the uncertainty associated with (1) model parameters and (2) model choice for the damage behavior, and then to further make predictions of fatigue degradation that rigorously incorporate different types of modeling uncertainty in a quantitative manner.

A set of five model classes pertaining to three families of damage mechanics models [23] (i.e., shearlag [24, 25], variational [26] and crack opening displacement (COD)[27, 28]) is chosen to represent the relation between the macro-scale stiffness reduction and the micro-scale damage, due to matrix-cracks. Damage mechanics models are preferred over other analytical approaches (e.g. continuum damage mechanics models or synergistic damage mechanics models [23]) due to their efficiency in relation to the assumptions adopted, and for being well connected with the physics of the underlying damage process. Moreover, these models have the ability to adapt to different systems (materials, loading conditions, etc.) without much training and 
furthermore, they can incorporate monitoring data in the Structural Health Monitoring (SHM) context.

Each candidate damage mechanics model is subsequently embedded into the modified Paris' law [29], that is used in this study to model the propagation forward in time of the matrix-cracks density. This two-level modeling approach results in a large number of uncertain parameters, leading to a computationally-intensive inference problem. To reduce the dimensionality of the problem without significantly altering the underlying uncertainty in the model output, a model input tuning is carried out by means of a Global Sensitivity Analysis (GSA) [30]. This allows to determine in advance the subset of parameters that are most "sensitive" to the model output uncertainty.

As a validation example, the proposed Bayesian framework is applied to damage data for matrix-crack density and stiffness reduction from a tension-tension fatigue experiment performed over a cross-ply CFRP laminate $[31,32]$. The results show that more complicated damage mechanics models not only involve more complicated analysis and adjustable parameters, but also do not yield higher probabilities in explaining the observed damage response. In this context, the evidence (also called as marginal likelihood) of each model class is revealed as a suitable measure to know the overall ability of the candidate model to predict the observed damage response, avoiding the extremes of over-fitting or under-fitting the data.

The paper is organized as follows. Section 2 discusses the theory behind fatigue damage in composites and presents the proposed methodology for fatigue damage modeling. In Section 3, the Bayesian inference framework for both model parameters and model classes is presented together with the problem of model parameterization using GSA. Section 4 is devoted to providing implementation details for conferring computational efficiency to the Bayesian inference problem. In particular, the computation of the likelihood function using the Graphics Processing Unit (GPU) is illustrated. In Section 5, the proposed framework is applied to a set of fatigue damage data to serve as an example. Finally Section 6 discusses the results and Section 7 provides concluding remarks.

\section{Fatigue damage modeling}

Typically, fatigue damage is perceived as a progressive or sudden change of the macro-scale mechanical properties, such as stiffness or strength, as a consequence of different fracture modes that evolve at the micro-scale along the lifespan of the structure [2]. In this research work, longitudinal stiffness loss is chosen as the macro-scale damage variable. In contrast to the strength, the stiffness can be measured through non-destructive methods during operation, which is of key importance for the model-updating approach proposed. At the micro-scale level, matrix micro-cracking [33] is selected as the dominant fracture mode for the early stage of damage accumulation.

Matrix cracks usually initiate from internal defects in $90^{\circ}$ plies during first loading cycles, and grow rapidly along fibers direction spanning the entire width of the specimen [33]. Continued loading leads to 
formation of new cracks between the already formed cracks thereby progressively increasing the matrix-cracks density of the ply until saturation. This saturated state, usually termed as characteristic damage state [1], is long recognized as a precursor of more severe fracture modes in adjacent plies, such as delamination and fiber breakage [34, 35], which may subsequently lead to the catastrophic failure of the laminate. In addition, matrix micro-cracking may itself constitute failure of the design when micro-cracks induced degradation in properties exceeds the predefined threshold.

To accurately represent the relation between this micro-scale damage mode and its manifestation through macro-scale properties, several families of micro-damage mechanics models can be found in the literature [23]. These models, that are grounded on first principles of admissible ply stress fields in presence of damage, can be roughly classified into 1) computational methods, 2) semi-analytical methods and 3) analytical methods. Among them, computational and semi-analytical methods have been shown to be promising approaches, however they are computationally intensive; hence a large number of repeated evaluations in a simulation-based inference procedure is computationally prohibitive. Therefore, we focus here on the set of analytical models to address the relationship between stiffness loss and micro-crack density. Three types of analytical models are considered: shear-lag models [24, 25], variational models [26], and crack opening displacement based models [27, 28].

Shear-lag models use one-dimensional approximations of the equilibrium stress field after cracking to derive expressions for stiffness properties of the cracked laminate. The main modeling assumption is basically that, in the position of matrix cracks, axial load is transferred to uncracked plies by the axial shear stresses at the interfaces. These models have received the most attention in the literature and, as a consequence, a vast number of modifications and extensions can be found. However, as stated by Talreja and Singh [23], all the one-dimensional shear-lag models are virtually identical, except for the choice of the shear-lag parameter, as explained later in this section.

Variational models are based on a two-dimensional approximation of the equilibrium stress field, that in contrast to shear-lag analysis, is obtained from the Principle of Minimum Complementary Energy [36, 37].

Finally, COD-based models use a 3-D homogenization procedure derived from the study of the average crack-face opening displacement of a single matrix crack as a function of the applied load, that can be calculated either analytically [27] or numerically [28, 38, 39]. While shear-lag and variational models are mostly applicable to cross-ply laminates (i.e. those with stacking sequence $\left[0 \frac{n_{0}}{2} / 90_{n_{90}} / 0_{\frac{n_{0}}{2}}\right]$, where $n_{0,90}=$ total number of plies at $0^{\circ}$ and $90^{\circ}$, respectively), COD-based models are applicable to general laminates with an arbitrary distribution of matrix cracks. The reader is referred to the recent work of Talreja and Singh [23] for a detailed overview of these models, but for the sake of clarity, the key formulation is appropriately reproduced here with a uniform notation. 


\subsection{Shear-lag and variational models}

Following the unifying formulation of Joffe and Varna [40], the effective longitudinal Young's modulus $E_{x}^{*}$ can be calculated in $\left[\phi_{\frac{n_{\phi}}{2}} / 90_{n_{90}} / \phi_{\frac{n_{\phi}}{2}}\right]$ laminates (where $\phi \in[-90,90]$ ) as a function of the crack-spacing in $90^{\circ}$ layers for both shear-lag and variational models as follows:

$$
E_{x}^{*}=\frac{E_{x, 0}}{1+a \frac{1}{2 l} R(\bar{l})}
$$

In the last equation, $E_{x, 0}$ is the longitudinal Young's modulus of the undamaged laminate, $\bar{l}=\frac{l}{t_{90}}$ is the half crack-spacing normalized with the $90^{\circ}$ sub-laminate thickness, $R(\bar{l})$ is the average stress perturbation function, and $a$ is a known function of laminate properties (defined in Appendix A). It should be noted that matrix-cracks density is usually defined as $\rho=\frac{1}{2 l}$, so that the normalized half crack-spacing $\bar{l}$ can be expressed as $\bar{l}=\frac{1}{2 \rho t_{90}}$. The function $R(\bar{l})$ takes different expressions depending on the approach considered:

$$
\begin{array}{ll}
R(\bar{l}) & =\frac{2}{\xi} \tanh (\xi \bar{l}) \\
R(\bar{l}) & =\frac{4 \alpha_{1} \alpha_{2}}{\alpha_{1}^{2}+\alpha_{2}^{2}} \frac{\cosh \left(2 \alpha_{1} \bar{l}\right)-\cos \left(2 \alpha_{2} \bar{l}\right)}{\alpha_{2} \sinh \left(2 \alpha_{1} \bar{l}\right)+\alpha_{1} \sin \left(2 \alpha_{2} \bar{l}\right)} \quad \text { (Shear-Lag) }
\end{array}
$$

where $\xi$ is the shear-lag parameter, and $\alpha_{1}, \alpha_{2}$ are known functions dependent on ply and laminate properties, as described in Appendix A.

Depending on the choice of parameter $\xi$, different shear-lag models can be obtained [23]. In this paper three candidate shear-lag models are selected, namely, the "classical" shear-lag model $[24,41]$ and two of its modifications: interlaminar shear-lag model [25], and bi-dimensional shear-lag model [42, 43]. The main difference between the classical approach and the interlaminar approach is that the later assumes that shear stresses develop within a resin rich region near the interfaces between adjacent plies, whose thickness $d_{0}$ and shear modulus $G_{m}$ are uncertain. The bi-dimensional approach is essentially equivalent to the classical approach except for that it introduces a minor correction to account for the Poisson's effect [23, 44]. See [23] for further discussion about shear-lag analysis.

The shear-lag parameter of each candidate model can be obtained as a function of ply and laminate properties (see Appendix A for nomenclature description), as follows:

$$
\begin{aligned}
\xi^{2} & =G_{23}\left(\frac{1}{E_{2}}+\frac{1}{\lambda E_{x}^{(\phi)}}\right) \\
\xi^{2} & =\frac{G_{m}}{d_{0}} t_{90}\left(\frac{1}{E_{2}}+\frac{1}{\lambda E_{x}^{(\phi)}}\right) \\
\xi^{2} & =\frac{\frac{1}{Q_{22}}+\frac{1}{\lambda Q_{x x}^{(\phi)}}}{\frac{1}{3 G_{23}}+\frac{\lambda}{3 G_{x z}^{(\phi)}}}
\end{aligned}
$$

where $Q_{22}$ in Equation 3c is the $(2,2)^{t h}$ element of the on-axis (local coordinates) ply stiffness matrix defined as $Q_{22}=\frac{E_{2}}{1-\nu_{12}^{2} E_{2} / E_{1}}$, and $Q_{x x}^{(\phi)}$ is the $(1,1)^{t h}$ element of the stiffness matrix of the $\left[\phi_{\frac{n_{\phi}}{2}}\right]$ sub-laminate. Given 
the ply stiffness matrix, which can be obtained from basic ply properties, the stiffness matrix of the total laminate or any sub-laminate can be obtained using the classical laminates plate theory [45].

\subsection{Crack opening displacement model}

For the COD-based model, the formulation of Gudmundson and Weilin [27] is adopted, which uses a closed-form expression for the average COD to derive expressions for the effective longitudinal Young's modulus in laminates with general layup, as:

$$
E_{x}^{*}=\frac{1}{\left(\left(\mathbf{S}_{0}\right)^{-1}-\sum_{k=1}^{n_{p}} \nu^{k} t^{k} \rho^{k}\left(\mathbf{A}^{k}\right)^{T} \sum_{i=1}^{n_{p}} \boldsymbol{\beta}^{i k} \mathbf{A}^{i}\right)_{(1,1)}^{-1}}
$$

In the last equation, $\mathbf{S}_{0}$ is the in-plane compliance matrix of the intact laminate, $n_{p}$ is the number of plies and, $\nu^{k}, t^{k}$ and $\rho^{k}$ stand for the volume fraction, the thickness and the matrix-cracks density of the $k^{t h}$ ply, respectively. $\mathbf{A}^{k}$ is a matrix determined by the compliance matrix and the unit normal vector on the crack surfaces of the $k^{t h}$ ply, and $\boldsymbol{\beta}^{i k}$ is a matrix associated with the average crack opening displacements and tractions of the surface of transverse cracks. Both are detailed in Appendix A. The subscript $(1,1)$ in Equation 4 denotes the first component of the resulting matrix.

\subsection{Damage propagation model}

Having identified the candidate models to express the relationship between the effective longitudinal Young's modulus and the micro-cracks density, the next step is to address the time evolution of the microcracks density. To this end, the previously explained micro-damage mechanics models are used to obtain the energy released per unit crack area due to the formation of a new crack between two existing cracks, denoted here as $G$. This energy, known as energy release rate (ERR), can be calculated as [46, 47]:

$$
G=\frac{\sigma_{x}^{2} h}{2 \rho t_{90}}\left(\frac{1}{E_{x}^{*}(2 \rho)}-\frac{1}{E_{x}^{*}(\rho)}\right)
$$

where $\sigma_{x}$ is the applied axial tension, and $h$ and $t_{90}$ are the laminate and $90^{\circ}$-sublaminate half-thickness, respectively. The result of the energy calculation is further introduced into the modified Paris' law [29] to obtain the evolution of matrix-cracks density as a function of fatigue cycle $n$, as shown below:

$$
\frac{d \rho}{d n}=A(\Delta G)^{\alpha}
$$

where $A$ and $\alpha$ are fitting parameters and $\Delta G$ is the increment in ERR for a specific stress amplitude: $\Delta G=G\left(\sigma_{x, \max }\right)-G\left(\sigma_{x, \min }\right)$. Due to the complexity of the expression for $\Delta G$, which involves the underlying micro-damage mechanics models for the computation of $E_{x}^{*}(\rho)$, a closed-form solution for Equation 6 is hard to obtain. To overcome this drawback, the resulting differential equation can be solved by approximating the derivative using "unit-time" finite differences, considering that damage evolves cycle-to-cycle as:

$$
\rho_{n}=\rho_{n-1}+A\left(\Delta G\left(\rho_{n-1}\right)\right)^{\alpha}
$$


To summarize, five micro-damage mechanics models, namely shear-lag $(\times 3)$, variational and COD models, are selected to compute $E_{x}^{*}(\rho)$, i.e. the relationship between the effective Young's modulus (macro-scale) and the matrix-cracks density (micro-scale). The evolution of matrix-cracks density is modeled by the modified Paris' law in Equation 7, that uses one of the candidate damage mechanics models to evaluate the increment in ERR. Therefore, for this study, five candidate models are considered to investigate the overall damage progression at both micro-scale and macro-scale.

\section{Bayesian methodology}

\subsection{Model class definition by stochastic embedding}

For the purpose of Bayesian model selection and parameter estimation, a probability-based description of the deterministic damage models described in Section 2 is needed. To this end, let us consider a candidate damage model that is defined by a deterministic relationship $\mathbf{g}=\mathbf{g}(\mathbf{u}, \mathbf{m}): \mathbb{R}^{N_{i}} \times \mathbb{R}^{N_{m}} \rightarrow \mathbb{R}^{N_{o}}$ between the model input $\mathbf{u} \in \mathbb{R}^{N_{i}}$ and the model output $\mathbf{g} \in \mathbb{R}^{N_{o}}$, given a set of $N_{m}$ uncertain model parameters $\mathbf{m} \in$ $\mathbb{R}^{N_{m}}$. This damage model can be "embedded" stochastically [22] by adding an error term $\mathbf{e}=\mathbf{e}(\mathbf{v}): \mathbb{R}^{N_{e}} \rightarrow$ $\mathbb{R}^{N_{o}}$ parameterized by $\mathbf{v} \in \mathbb{R}^{N_{e}}$, that represents the difference between the measured output $\mathbf{y} \in \mathbb{R}^{N_{o}}$ and the model output $\mathrm{g}$, as follows:

$$
\underbrace{\mathbf{y}}_{\text {measurements }}=\underbrace{\mathbf{g}(\mathbf{u}, \mathbf{m})}_{\text {model }}+\underbrace{\mathbf{e}(\mathbf{v})}_{\text {error }}
$$

In the last equation, it is assumed that both the measurement and model errors are subsumed into the error term $\mathbf{e}(\mathbf{v})$. Such assumption is commonly adopted when the measurement error is negligible as compared to the model error, or when an independent study about the measurement error is not available. In addition, the set $\mathbf{m}$ of model parameters is augmented with the set $\mathbf{v}$ of error parameters, resulting in a set of model parameters defined as $\boldsymbol{\theta}=\{\mathbf{m}, \mathbf{v}\} \in \mathbf{\Theta} \subset \mathbb{R}^{d=N_{m}+N_{e}}$. This set of parameters is further updated through Bayes' Theorem, as explained in Section 3.3.

It should be noted here that the probability model chosen for the error term $\mathbf{e}$ in Equation 8 determines the probability model for the observed output $\mathbf{y}$. For example, if the error term is assumed to be modeled as a Gaussian distribution, i.e. $\mathbf{e} \sim \mathcal{N}\left(\boldsymbol{\mu}_{\mathbf{e}}, \boldsymbol{\Sigma}_{\mathbf{e}}\right)$, then the observed output $\mathbf{y}$ will be also distributed as a Gaussian, as follows:

$$
\mathbf{e}=\mathbf{y}-\mathbf{g}(\mathbf{u}, \boldsymbol{\theta}) \sim \mathcal{N}\left(\boldsymbol{\mu}_{\boldsymbol{e}}, \boldsymbol{\Sigma}_{\mathbf{e}}\right) \Longrightarrow \mathbf{y} \sim \mathcal{N}\left(\mathbf{g}(\mathbf{u}, \boldsymbol{\theta})+\boldsymbol{\mu}_{\mathbf{e}}, \boldsymbol{\Sigma}_{\mathbf{e}}\right)
$$

where $\boldsymbol{\mu}_{\mathrm{e}} \in \mathbb{R}^{N_{o}}$ is a systematic bias between model output and observations, and $\boldsymbol{\Sigma}_{\mathbf{e}} \in \mathbb{R}^{N_{o} \times N_{o}}$ is a covariance matrix. This assumption is supported by the Principle of Maximum Information Entropy (PMIE) [21, 48], which enables a rational way to establish a probability model for the error term such that it produces the largest uncertainty (largest Shannon entropy). Thus, by adopting this assumption, a 
stochastic model of damage can be defined from any deterministic model as

$$
p(\mathbf{y} \mid \mathbf{u}, \boldsymbol{\theta}, \mathcal{M})=\left((2 \pi)^{N_{o}}\left|\boldsymbol{\Sigma}_{\mathbf{e}}\right|\right)^{-\frac{1}{2}} \exp \left(-\frac{1}{2}(\mathbf{y}-\overline{\mathbf{y}})^{T} \boldsymbol{\Sigma}_{\mathbf{e}}^{-1}(\mathbf{y}-\overline{\mathbf{y}})\right)
$$

where $\overline{\mathbf{y}}=\mathbf{g}(\mathbf{u}, \boldsymbol{\theta})+\boldsymbol{\mu}_{\mathbf{e}}$ denotes the mean output of the stochastic model and $\mathcal{M}$ represents the candidate Bayesian model class, defined by the stochastic model of damage and the prior probability density function (PDF) of model parameters, $p(\boldsymbol{\theta} \mid \mathcal{M})$. This prior PDF represents the initial relative plausibility of parameters $\boldsymbol{\theta}$ before the information from measurements is incorporated through Bayesian updating, as explained further below.

\subsection{Stochastic embedding for deterministic damage models}

As discussed in Section 2, the progression of fatigue damage in composites is studied at every fatigue cycle $n$ by focusing on two of its manifestations: the matrix-cracks density, $\rho_{n}$, and the normalized effective stiffness, defined as $D_{n}=\frac{E_{x}^{*}}{E_{x, 0}}$. Then, according to Equation 8, the overall system response can be represented by:

$$
\begin{gathered}
\rho_{n}=\underbrace{g_{1}\left(\rho_{n-1}, \mathbf{u}, \boldsymbol{\theta}\right)}_{\text {Equation } 7}+e_{1} \\
D_{n}=\underbrace{g_{2}\left(\rho_{n}, \mathbf{u}, \boldsymbol{\theta}\right)}_{\text {Equations 1 \& } 4}+e_{2}
\end{gathered}
$$

where subscripts 1 and 2 denote the corresponding damage subsystems: matrix-crack density and normalized effective stiffness, respectively.

From Equations 11a and 11b, the three main elements defining the stochastic damage model in Equation 10 can be identified: (1) the observations $\mathbf{y}_{n}=\left(\rho_{n}, D_{n}\right) \in \mathbb{R}^{2},(2)$ the model output $\mathbf{g}=\left(g_{1}, g_{2}\right) \in$ $\mathbb{R}^{2}$, and (3) the corresponding error term $\mathbf{e}=\left(e_{1}, e_{2}\right) \in \mathbb{R}^{2}$. A key concept here is the consideration of errors $e_{1}$ and $e_{2}$ as stochastically independent (i.e., not correlated) a priori, even though the models corresponding to the damage subsystems $g_{1}$ and $g_{2}$ are mathematically related, as shown in Section 2 . This means that the covariance operator $\boldsymbol{\Sigma}_{\mathbf{e}}$ is a diagonal matrix, i.e. $\boldsymbol{\Sigma}_{\mathbf{e}}=\operatorname{diag}\left(\sigma_{e_{1}}^{2}, \sigma_{e_{2}}^{2}\right)$; therefore, Equation 10 can be readily expressed as a product of univariate Gaussians, as follows:

$$
p\left(\mathbf{y}_{n} \mid \mathbf{u}, \boldsymbol{\theta}, \mathcal{M}\right)=p\left(\rho_{n} \mid \rho_{n-1}, \mathbf{u}, \boldsymbol{\theta}, \mathcal{M}\right) p\left(D_{n} \mid \rho_{n}, \mathbf{u}, \boldsymbol{\theta}, \mathcal{M}\right)
$$

where

$$
\begin{gathered}
p\left(\rho_{n} \mid \rho_{n-1}, \mathbf{u}, \boldsymbol{\theta}, \mathcal{M}\right)=\mathcal{N}\left(g_{1}\left(\rho_{n-1}, \mathbf{u}, \boldsymbol{\theta}\right)+\mu_{e_{1}}, \sigma_{e_{1}}^{2}\right) \\
p\left(D_{n} \mid \rho_{n}, \mathbf{u}, \boldsymbol{\theta}, \mathcal{M}\right)=\mathcal{N}\left(g_{2}\left(\rho_{n}, \mathbf{u}, \boldsymbol{\theta}\right)+\mu_{e_{2}}, \sigma_{e_{2}}^{2}\right)
\end{gathered}
$$

and $\left(\mu_{e_{1}}, \sigma_{e_{1}}\right)$ and $\left(\mu_{e_{2}}, \sigma_{e_{2}}\right)$ are the parameters (mean and standard deviation) of the error terms $e_{1}$ and $e_{2}$, respectively; i.e., $\mathbf{v}=\left\{\mu_{e_{1}}, \sigma_{e_{1}}, \mu_{e_{2}}, \sigma_{e_{2}}\right\}$. 
The same assumption of stochastic independency made for error parameters $\mathbf{v}$ can also be adopted for the rest of model parameters in $\boldsymbol{\theta}$. Under this assumption, the prior PDF of model parameters is defined as the unconditional product of the individual priors $p\left(\theta_{i} \mid \mathcal{M}\right), i=1, \ldots, d$; i.e., $p(\boldsymbol{\theta} \mid \mathcal{M})=\prod_{i=1}^{d} p\left(\theta_{i} \mid \mathcal{M}\right)$. Note that this assumption is not an assertion that no correlations actually exist in model parameters, but is only a description of the available prior information about such correlations. If they existed, they would become apparent after Bayesian updating and therefore, they would be considered in subsequent forward model simulations.

\subsection{Bayesian model updating}

The focus of Bayesian model updating is to obtain the posterior PDF of model parameters $\boldsymbol{\theta}$ over the set $\Theta \subset \mathbb{R}^{d}$ of possible values in the model class $\mathcal{M}$, based on the information of system response contained in data $\mathcal{D}$. It can be accomplished using Bayes' Theorem, as follows:

$$
\underbrace{p(\boldsymbol{\theta} \mid \mathcal{D}, \mathcal{M})}_{\text {posterior }}=c^{-1} p(\mathcal{D} \mid \boldsymbol{\theta}, \mathcal{M}) \underbrace{p(\boldsymbol{\theta} \mid \mathcal{M})}_{\text {prior }}
$$

where $c$ is a normalizing constant so that $p(\boldsymbol{\theta} \mid \mathcal{D}, \mathcal{M})$ represents a valid PDF, as:

$$
\int_{\Theta} p(\boldsymbol{\theta} \mid \mathcal{D}, \mathcal{M}) d \boldsymbol{\theta}=c^{-1} \int_{\Theta} p(\mathcal{D} \mid \boldsymbol{\theta}, \mathcal{M}) p(\boldsymbol{\theta} \mid \mathcal{M}) \mathrm{d} \boldsymbol{\theta}=1
$$

Here, the $\operatorname{PDF} p(\mathcal{D} \mid \boldsymbol{\theta}, \mathcal{M})$ is known as the likelihood function, and provides a measure of how well the model specified by $\boldsymbol{\theta}$ within the model class $\mathcal{M}$ predicts the observed system response $\mathcal{D}$.

\subsection{Formulation of the likelihood function from physics-based models}

In this study, data $\mathcal{D}$ consists of an experimental sequence of $N$ fatigue damage measurements $\mathcal{D}=$ $\left\{\mathbf{y}_{1}, \ldots, \mathbf{y}_{n}, \ldots, \mathbf{y}_{N}\right\}$ defined over a set of fatigue cycles $\mathcal{T}=\{1, \ldots, n, \ldots, N\} \subset \mathbb{N}$, where $\mathbf{y}_{n}=\left(\rho_{n}, D_{n}\right)$. The likelihood function is then computed as the probability of predicting the experimental sequence $\mathcal{D}$ by the stochastic model defined in Equation 12 under the parameterization specified by $\boldsymbol{\theta}$ within the model class $\mathcal{M}$, as follows:

$$
p(\mathcal{D} \mid \boldsymbol{\theta}, \mathcal{M})=\prod_{n=1}^{N} p\left(\mathbf{y}_{n} \mid \mathbf{u}, \boldsymbol{\theta}, \mathcal{M}\right)
$$

By substituting Equation 12 into Equation 16, the likelihood function can be finally expressed as:

$$
p(\mathcal{D} \mid \boldsymbol{\theta}, \mathcal{M})=\prod_{n=1}^{N} p\left(D_{n} \mid \rho_{n}, \mathbf{u}, \boldsymbol{\theta}, \mathcal{M}\right) p\left(\rho_{n} \mid \rho_{n-1}, \mathbf{u}, \boldsymbol{\theta}, \mathcal{M}\right)
$$

Note that when data are available over a set of non-regularly scheduled cycles $\mathcal{T}_{D}=\left\{n_{k}, n_{l}, \ldots, n_{N}\right\}$, $\mathcal{T}_{D} \subset \mathcal{T}$, the likelihood function defined in Equation 17 cannot be evaluated. This is due to the "one-step" description of the matrix-cracks evolution model, as is defined in Equation 11a. To overcome this drawback, which is usual in fatigue testing, the Total Probability Theorem can be applied to bridge the missing damage 
path growth between two non-subsequent measurements of matrix-cracks density. For example, for general cycles $n_{k}$ and $n_{l}$, such that $n_{l}=n_{k}+\Delta n$ with $\Delta n \in \mathbb{N} \geqslant 1$, the probability $p\left(\rho_{n_{l}} \mid \rho_{n_{k}}, \mathbf{u}, \boldsymbol{\theta}, \mathcal{M}\right)$ in Equation 17 can be calculated as:

$$
p\left(\rho_{n_{l}} \mid \rho_{n_{k}}, \boldsymbol{\theta}\right)=\int p\left(\rho_{n_{l}} \mid \Gamma_{n_{k}}^{n_{l}}, \rho_{n_{k}}, \boldsymbol{\theta}\right) p\left(\Gamma_{n_{k}}^{n_{l}} \mid \rho_{n_{k}}, \boldsymbol{\theta}\right) d \Gamma_{n_{k}}^{n_{l}}
$$

where $\Gamma_{n_{k}}^{n_{l}}$ represents the missing damage sequence between the observed data $\rho_{n_{k}}$ and $\rho_{n_{l}}$, i.e. $\Gamma_{n_{k}}^{n_{l}}=$ $\left\{\rho_{n_{k}+1}, \rho_{n_{k}+2}, \ldots, \rho_{n_{l}-1}\right\}$. Note that, for the sake of clarity, the conditioning on model class $\mathcal{M}$ and model inputs $\mathbf{u}$ is dropped. The high dimensional probability integral in Equation 18 can be readily estimated as a mathematical expectation using the direct Monte Carlo (MC) method, as:

$$
p\left(\rho_{n_{l}} \mid \rho_{n_{k}}, \boldsymbol{\theta}\right) \approx \frac{1}{T} \sum_{i=1}^{T} p\left(\rho_{n_{l}} \mid \tilde{\Gamma}_{n_{k}}^{n_{l},(i)}, \rho_{n_{k}}, \boldsymbol{\theta}\right)
$$

where $\tilde{\Gamma}_{n_{k}}^{n_{l},(i)}=\left\{\tilde{\rho}_{n_{k}+1}^{(i)}, \tilde{\rho}_{n_{k}+2}^{(i)}, \ldots, \tilde{\rho}_{n_{l}-1}^{(i)}\right\}$ is the $i^{t h}$ simulated sequence of damage growth between cycles $n_{k}$ and $n_{l}, i=1, \ldots, T$. It can be obtained by conditional sampling from the stochastic matrix-cracks evolution model given in Equation 13a: first sample $\tilde{\rho}_{n_{k}+1}^{(i)}$ using the aforementioned evolution model conditional on the initial observed state $\rho_{n_{k}}$, i.e. $\tilde{\rho}_{n_{k}+1}^{(i)} \sim p\left(\cdot \mid \rho_{n_{k}}, \boldsymbol{\theta}\right)$; then sample the succeeding state conditional on the previous sample, i.e. $\tilde{\rho}_{n_{k}+2}^{(i)} \sim p\left(\cdot \mid \tilde{\rho}_{n_{k}+1}^{(i)}, \boldsymbol{\theta}\right)$; finally, repeat the same process until the final damage state in $\tilde{\Gamma}_{n_{k}}^{n_{l},(i)}$ is reached.

By the Markovian type of evolution of the matrix-cracks density, inherited from the definition of the crack evolution model in Equation 11a, the probability of any future damage state is conditionally independent of the past history given the immediately previous state, thus $p\left(\rho_{n_{l}} \mid \Gamma_{n_{k}}^{n_{l}}, \boldsymbol{\theta}\right)=p\left(\rho_{n_{l}} \mid \rho_{n_{l}-1}, \boldsymbol{\theta}\right)$. Then Equation 19 can be rewritten as:

$$
p\left(\rho_{n_{l}} \mid \rho_{n_{k}}, \boldsymbol{\theta}\right) \approx \frac{1}{T} \sum_{i=1}^{T} p\left(\rho_{n_{l}} \mid \tilde{\rho}_{n_{l}-1}^{(i)}, \boldsymbol{\theta}\right)
$$

where $\tilde{\rho}_{n_{l}-1}^{(i)}$ is the last damage state in the simulated sequence $\tilde{\Gamma}_{n_{k}}^{n_{l},(i)}$. The probability estimate obtained from Equation 20 is further inserted in Equation 17 to calculate the overall likelihood of the damage model specified by $\boldsymbol{\theta}$ in model class $\mathcal{M}$. Figure A.1 provides further clarification about the calculation of the likelihood along with details for its computational implementation, as will be shown further below.

\subsection{Parameter selection by Global Sensitivity Analysis}

The Bayesian approach to model parameter estimation and model class selection involves an inference problem defined over a multi-dimensional parameter space $\boldsymbol{\Theta} \subset \mathbb{R}^{d}$. It is clear that the higher $d$, the higher the complexity and computational cost of the updating process. At the same time, adopting a predetermined set of model parameters may lead to an unjustified uncertainty reduction [49]. Sankararaman et al. [16] addressed this issue for fatigue crack growth prediction in metals, where GSA was used to select the model parameters that were further updated using Bayes' Theorem. A similar approach has recently been adopted 
by Gobbato et al. [19] for fatigue crack growth prediction in metals by using partial-derivatives for sensitivity analysis. In this paper, a variance-based approach for GSA is adopted following the approach by [16], i.e. simplify the model parameterization by identifying the subset of parameters that can be fixed at any given value (e.g., the mean or nominal value) of their range of variation without affecting the uncertainty of the model output.

For the sake of illustration, let consider the model $\mathbf{g}: \mathbf{g}(\psi)$ defined in Section 3.1 as a function of the set of parameters $\boldsymbol{\psi}=\{\mathbf{u}, \mathbf{m}\}=\left\{\psi_{1}, \ldots, \psi_{i}, \ldots, \psi_{N_{p}}\right\} \subset \mathbb{R}^{N_{p}=N_{i}+N_{m}}$. Each component $\psi_{i}$ is defined over a non-null range of variation or uncertainty determined by the prior PDF. The goal is to identify $\mathbf{u} \subset \boldsymbol{\psi}$, as the subset of non-influential parameters. The necessary and sufficient condition for parameter $\psi_{i}$ to be noninfluential is that $S_{i}^{T}=0$ [49], where $S_{i}^{T}$ is the total effects index of parameter $\psi_{i}$, which can be computed as [30]:

$$
S_{i}^{T}=\frac{\mathbb{E}_{\psi_{\sim i}}\left(V_{\psi_{i}}\left(\mathbf{g} \mid \psi_{\sim i}\right)\right)}{V(\mathbf{g})}
$$

with $S_{i}^{T} \in[0,1]$ and $\sum_{i=1}^{N_{p}} S_{i}^{T} \geqslant 1$. The numerator of the right hand side of Equation 21 can be evaluated using double-loop MC sampling, although single-loop MC sampling approaches have also been discussed in the literature [30]. In the inner loop, the conditional variance $V_{\psi_{i}}\left(\mathbf{g} \mid \psi_{\sim i}\right)$ is calculated by evaluating the model considering random variations in $\psi_{i}$, when the parameters other than $\psi_{i}$ (denoted by $\psi_{\sim i}$ ) are fixed at a random value sampled from the associated prior PDFs. The outer loop considers random variations in $\psi_{\sim i}$ and computes the expectation of the aforementioned variance. Finally, the result is divided by $V(\mathbf{g})$, the unconditional variance of the model response, which can be readily obtained by evaluating the model using samples from the joint prior PDF of the complete set parameters $\boldsymbol{\psi}$. As stated before, the lower the total effects index of a particular parameter $\psi_{i}$, the smaller its influence for Bayesian updating. Therefore, parameters with low sensitivity are left out of the updating procedure, thereby reducing the dimensionality of the problem.

This procedure is applied for each candidate damage model and, as a result, a subset $\mathbf{m} \subseteq \boldsymbol{\psi}$ of model parameters arises for each model class. The rest of non-influential parameters are then used as deterministic input parameters $\mathbf{u}$, hence they can be fixed anywhere within their range of variation. As stated in Section 3.1 , the vector $\mathbf{m}$ of model parameters is augmented with the set of error parameters $\mathbf{v}$, resulting in a set of model parameters for Bayesian updating defined by $\boldsymbol{\theta}=\{\mathbf{m}, \mathbf{v}\} \in \boldsymbol{\Theta} \subset \mathbb{R}^{d=N_{m}+N_{e}}$.

\subsection{Bayesian model-class selection}

The probabilistic approach for model class selection is motivated by the fact that the model itself may not necessarily reproduce the observed system, but it is just an approximation [21, 50]. The goal is to use the available damage data $\mathcal{D}$ to asses the probability of the $j^{\text {th }}$ model class within the set $\mathbf{M}=\left\{\mathcal{M}_{1}, \ldots, \mathcal{M}_{j}, \ldots, \mathcal{M}_{N_{M}}\right\}$ of candidate classes for representing the system. Probabilities are obtained 
using Bayes' Theorem at the model class level as:

$$
p\left(\mathcal{M}_{j} \mid \mathcal{D}, \mathbf{M}\right)=\frac{p\left(\mathcal{D} \mid \mathcal{M}_{j}\right) p\left(\mathcal{M}_{j} \mid \mathbf{M}\right)}{\sum_{i=1}^{N_{M}} p\left(\mathcal{D} \mid \mathcal{M}_{i}\right) p\left(\mathcal{M}_{i} \mid \mathbf{M}\right)}
$$

where $p\left(\mathcal{M}_{j} \mid \mathbf{M}\right)$ is the prior probability of each model class, that expresses the initial modeler's judgement on the relative degree of belief on $\mathcal{M}_{j} \in \mathbf{M}$. The factor $p\left(\mathcal{D} \mid \mathcal{M}_{j}\right)$ is the evidence (or marginal likelihood) for the model class $\mathcal{M}_{j}$, and expresses how likely the observed data are reproduced if model class $\mathcal{M}_{j}$ is adopted. Note that the evidence is equal to the normalizing constant in establishing the posterior PDF in Equation 14, so that it can be obtained as

$$
p\left(\mathcal{D} \mid \mathcal{M}_{j}\right)=\int_{\Theta} p\left(\mathcal{D} \mid \boldsymbol{\theta}, \mathcal{M}_{j}\right) p\left(\boldsymbol{\theta} \mid \mathcal{M}_{j}\right) \mathrm{d} \boldsymbol{\theta}
$$

However the evaluation of the last multi-dimensional integral is nontrivial except for some cases where the Laplace's method of asymptotic approximation can be used [51]. In this work, a recent technique based on samples from the posterior is adopted to numerically solve this integral [52].

\subsection{Information-theory approach to model-class selection}

In addition to compute the plausibility of a particular model class, it is also of much interest to know the quality of the data-fit in relation to the complexity of such model class, i.e., the amount of information extracted from data, to avoid the extremes of over-fitting or under-fitting. A common principle enunciated is that, if data are explained equally well by two models, then the "simpler" one should be preferred (often referred to as Ockham's razor [21]). To tackle this problem, Muto and Beck [53] proposed an informationtheoretic interpretation of the evidence for a model class, as follows:

$$
\begin{aligned}
\log p\left(\mathcal{D} \mid \mathcal{M}_{j}\right) & =\int_{\boldsymbol{\Theta}}\left[\log p\left(\mathcal{D} \mid \boldsymbol{\theta}, \mathcal{M}_{j}\right)\right] p\left(\boldsymbol{\theta} \mid \mathcal{D}, \mathcal{M}_{j}\right) d \boldsymbol{\theta}-\int_{\boldsymbol{\Theta}}\left[\log \frac{p\left(\boldsymbol{\theta} \mid \mathcal{D}, \mathcal{M}_{j}\right)}{p\left(\boldsymbol{\theta} \mid \mathcal{M}_{j}\right)}\right] p\left(\boldsymbol{\theta} \mid \mathcal{D}, \mathcal{M}_{j}\right) d \boldsymbol{\theta} \\
& =\mathbb{E}\left[\log p\left(\mathcal{D} \mid \boldsymbol{\theta}, \mathcal{M}_{j}\right)\right]-\mathbb{E}\left[\log \frac{p\left(\boldsymbol{\theta} \mid \mathcal{D}, \mathcal{M}_{j}\right)}{p\left(\boldsymbol{\theta} \mid \mathcal{M}_{j}\right)}\right]
\end{aligned}
$$

where $\mathbb{E}$ is the expectation respect to the posterior $p\left(\boldsymbol{\theta} \mid \mathcal{D}, \mathcal{M}_{j}\right)$.

The first term of Equation 24 is a measure of the average goodness of fit (AGF) of the model class $\mathcal{M}_{j}$ to the data $\mathcal{D}$. The second term is the relative entropy between the posterior and the prior PDFs, which measures the "difference" between those PDFs [54]. This term determines the expected information gained (EIG) about the model class $\mathcal{M}_{j}$ from the data and it is, by definition, always non-negative. Therefore, the log-evidence of a model class is comprised of a data-fit term and a term that provides a penalty against more complex model classes, that are those that extract more information from the data to update their prior information. It allows us to find a correct trade-off between fitting accuracy and model complexity, and gives an intuitive understanding of why the computation of evidence automatically enforces a quantitative expression of the Principle of Model Parsimony or Ockham's razor [21]. 


\section{Implementation details}

The proposed Bayesian methodology involves the evaluation of multi-dimensional integrals of the type occurring in Equations 14, 18 and 23. The direct Monte Carlo method may be used to estimate these integrals, although with some known drawbacks of inefficiency and instability [22]. As explained in Section 4.1, Markov Chain Monte Carlo (MCMC) methods [55] can deal with these difficulties, however they are not straightforward to implement. In particular, for this research work, there are some implementation issues such as those shown in Section 4.2, that must be conveniently treated to avoid heavy computation.

\subsection{Metropolis-Hastings algorithm for Bayesian model updating}

The goal of MCMC methods in Bayesian updating is to generate parameter samples which are distributed according to the target posterior $\operatorname{PDF} p\left(\boldsymbol{\theta} \mid \mathcal{D}, \mathcal{M}_{j}\right)$. A particular advantage of these methods is that they only require specification of the target up to a constant, which avoids the calculation of the normalization constant in Equation 14. Several MCMC algorithms have been proposed in the literature, such as the Metropolis-Hastings (M-H) and Gibbs Sampler, among others [55].

In this work, the $\mathrm{M}-\mathrm{H}$ algorithm $[56,57]$ is used for its versatility and implementation simplicity. This algorithm generates samples from a specially constructed Markov chain whose stationary distribution is the posterior PDF. By sampling a candidate model parameter $\boldsymbol{\theta}^{\prime}$ from a proposal distribution $q\left(\boldsymbol{\theta}^{\prime} \mid \boldsymbol{\theta}^{(\zeta)}\right)$, the M-H obtains the state of the chain at $\zeta+1$, given the state at $\zeta$, specified by $\boldsymbol{\theta}^{(\zeta)}$. The candidate parameter $\boldsymbol{\theta}^{\prime}$ is accepted (i.e. $\boldsymbol{\theta}^{(\zeta+1)}=\boldsymbol{\theta}^{\prime}$ ) with probability $\min \{1, r\}$, and rejected $\left(\boldsymbol{\theta}^{(\zeta+1)}=\boldsymbol{\theta}^{(\zeta)}\right)$ with the remaining probability $1-\min \{1, r\}$, where:

$$
r=\frac{p\left(\mathcal{D} \mid \boldsymbol{\theta}^{\prime}, \mathcal{M}_{j}\right) p\left(\boldsymbol{\theta}^{\prime} \mid \mathcal{M}_{j}\right) q\left(\boldsymbol{\theta}^{(\zeta)} \mid \boldsymbol{\theta}^{\prime}\right)}{p\left(\mathcal{D} \mid \boldsymbol{\theta}^{(\zeta)}, \mathcal{M}_{j}\right) p\left(\boldsymbol{\theta}^{(\zeta)} \mid \mathcal{M}_{j}\right) q\left(\boldsymbol{\theta}^{\prime} \mid \boldsymbol{\theta}^{(\zeta)}\right)}
$$

The process is repeated until $N_{s}$ samples have been generated. More details about this algorithm are provided in Section 5.2 in the context of the case study presented in this article.

\subsection{GPU acceleration of $M-H$ algorithm}

It can be observed from Equation 25 that each MCMC step requires the evaluation of the overall likelihood function $p\left(\mathcal{D} \mid \boldsymbol{\theta}, \mathcal{M}_{j}\right)$, which (recall Equation 17) involves the evaluation of multi-dimensional integrals such as those defined by Equation 18. The MC method is applied to numerically solve these integrals, as shown in Equation 19, however it requires the simulation of $T$ (large enough) damage growth sequences $\left\{\tilde{\Gamma}_{n_{k}}^{n_{l},(i)}\right\}_{i=1}^{T}$, which increases the computational complexity dramatically.

To speed up these computations, the evaluation of the heaviest part of the likelihood is carried out in parallel on the Graphics Processing Unit (GPU), utilizing the Compute Unified Device Architecture (CUDA) code from NVIDIA using Matlabß. Figure A.1 provides a scheme of the M-H algorithm implementation 
using the GPU. Observe that the simulation of the $T$ damage growth sequences, $\left\{\tilde{\Gamma}_{n_{k}}^{n_{l},(i)}\right\}_{i=1}^{T}$, together with the evaluation of the PDFs $\left\{p\left(\rho_{n_{l}} \mid \tilde{\rho}_{n_{l}-1}^{(i)}, \boldsymbol{\theta}\right)\right\}_{i=1}^{T}$ (recall Equation 20), are performed using the GPU. The summation of the individual probability density values of Equation 20 together with the remaining part of the likelihood function are handled by the CPU. The CPU also takes charge of the rest of the M-H steps. An averaged speed-up factor of up to 900, compared to an equivalent serial computation on the CPU, is observed while updating each model class using a GPU NVIDIA GeForce GTX 680 (1536 CUDA cores) and a $3.2 \mathrm{GHz}$ CPU system.

\section{Case study}

In order to investigate the performance of the proposed approach, SHM data obtained from a set of carefully designed run-to-failure fatigue experiments were used. Both stiffness data and NDE measurements of internal damage, such as micro-crack density and delamination area, were periodically measured during the fatigue test [32]. Torayca T700G uni-directional carbon-prepreg material was used for $15.24 \mathrm{~cm} \times 25.4 \mathrm{~cm}$ coupons with dogbone geometry and $\left[0_{2} / 90_{4}\right]_{\mathrm{S}}$ stacking sequence. The mechanical properties of such coupons are listed in Table A.2. A notch $(5.1 \mathrm{~mm} \times 19.3 \mathrm{~mm})$ was created in the central section of these coupons to induce damage modes others than matrix-cracks, such as delamination, thereby introducing additional sources of uncertainty and then demonstrating the proposed framework under more realistic conditions. Fatigue tests were conducted under load-controlled tension-tension cyclic loading, with a maximum applied load of $31.13 \mathrm{KN}$, a frequency $f=5 \mathrm{~Hz}$, and a stress ratio $R=0.14$ (relation between the minimum and maximum stress for each cycle). Monitoring data were collected from a network of 12 piezoelectric (PZT) sensors using Lamb wave signals and three triaxial strain-gages. Additionally, periodic X-rays were taken to visualize and characterize subsurface damage features, in particular, the micro-crack density. This information was then used to develop a mapping between PZT raw signals and the observed micro-crack density, as reported in Larrosa and Chang [58]. Damage data used in this example correspond to laminate L1S19 in [31], (see a summary in Table A.1). The experimental set-up is shown in Figure A.2. More details about these tests are reported in the Composite dataset, NASA Ames Prognostics Data Repository [31].

\subsection{Definition of model classes}

Five model classes are considered for the inference, so $N_{M}=5$ in Equation 22. Three of them are based on the shear-lag analysis $\left(\mathcal{M}_{1}\right.$ : classical, $\mathcal{M}_{2}$ : bidimensional, $\mathcal{M}_{3}$ : interlaminar $)$, and the rest are based on the variational $\left(\mathcal{M}_{4}\right)$ and $\operatorname{COD}\left(\mathcal{M}_{5}\right)$ approaches, respectively. Observe that model classes $\mathcal{M}_{1}$ to $\mathcal{M}_{5}$ have an increasing level of analysis complexity. 


\subsubsection{Probabilistic representation of parameters}

Table A.2 lists the prior information (namely, the prior PDF) of model parameters, including the fitting parameters in the modified Paris' law and the error parameters. Since mechanical and fitting parameters $\mathbf{m} \subset \boldsymbol{\theta}$ are non-negative, their associated prior information can be modeled as a lognormal distribution, i.e., $p\left(\theta_{i} \mid \mathcal{M}\right)=\mathcal{L N}\left(\mu_{\theta_{i}}, \sigma_{\theta_{i}}\right), i=1, \ldots, N_{m}$, with $\mu_{\theta_{i}}=\ln \bar{\theta}_{i}$, being $\bar{\theta}_{i}$ the nominal value of $\theta_{i} \in \boldsymbol{\theta}$ (e.g., the mean), and $\sigma_{\theta_{i}}$ the shape parameter of the lognormal distribution. Both, the nominal values and shape parameters are listed in Table A.2. For the error parameters $\mathbf{v} \subset \boldsymbol{\theta}$, a uniform distribution defined over a sufficiently-large predefined interval (conservatively chosen after some initial test runs) is selected as the prior PDF. This choice is preferred instead of the common choice of using "non-informative" priors [21] (e.g., a uniform distribution over a very large interval), which would better represent our prior state of information about these parameters. While such a choice may not significantly influence the posterior PDF of parameters, the use of excessively diffuse priors may lead to a markedly high information gain from data, which would induce a bias in the model-class selection problem [22]. Note also that our assumptions about the prior PDFs given in Table A.2 can be conveniently updated if more information is available, for example from expert judgement.

\subsubsection{Model parameterization}

As explained in Section 3.5, GSA aims at selecting the influential model parameters among the mechanical and fitting parameters listed in Table A.2, which are further updated using Bayes' Theorem. To evaluate Equation 21, a double-loop Monte Carlo algorithm is implemented using $10^{3}$ and $10^{4}$ samples for the inner and outer loops, respectively. Results from GSA are shown in Figure A.3 for model classes $\mathcal{M}_{1}$ to $\mathcal{M}_{5}$.

To obtain the set of parameters to be updated by Bayes' Theorem, namely $\boldsymbol{\theta}$, the subset of error parameters $\mathbf{v}=\left\{\mu_{e_{1}}, \sigma_{e_{1}}, \mu_{e_{2}}, \sigma_{e_{2}}\right\}$ is added to $\mathbf{m}$, the subset of "sensitive" parameters identified by GSA. To serve as an example, the model parameter vector for the shear-lag model class $\mathcal{M}_{1}$ would be $\boldsymbol{\theta}=$ $\left\{\alpha, E_{1}, E_{2}, t, \mu_{e_{1}}, \sigma_{e_{1}}, \mu_{e_{2}}, \sigma_{e_{2}}\right\}$. The rest of parameters would define the set of constant parameters $\mathbf{u}$, i.e., $\mathbf{u}=\left\{A, G_{12}, G_{23}, \nu_{12}\right\}$, which may be represented by their nominal values (specified in Table A.2) without any associated uncertainty. The set of model parameters for each model class is found in Table A.4.

It is important to remark here that, for all model classes, the influence of the modified Paris' law fitting parameter $A$ is found to be insignificant as compared to the sensitivity of most mechanical parameters (see Figure A.3). This is especially convenient given that mechanical parameters are usually benefited from less uncertain prior information. In other words, mechanical parameters are likely to capture much less information from data as compared to fitting parameters, which (recall Equation 24) leads to model parameterizations with lower associated EIG terms. As discussed further below, this results in more robust model classes, i.e., with a lower dependence on the details of data.

Finally, in view of Figure A.3, a possible question that arises is that what would be the choice if any of the 
candidate parameters $\psi_{i}$ becomes influential but only for a certain stage of the process (e.g., the initial or the final stage). In this case, the authors' recommendation is to consider $\psi_{i}$ as influential for all the process since the unnecessary increase of model output uncertainty incurred when considering $\psi_{i}$ as influential (when it is actually non-influential) is by definition null or at least negligible [30]. As stated before, a non-influential parameter can be fixed anywhere in their range of variation without effect in the model output uncertainty.

\subsection{Model parameter updating}

As presented in Section 3.3, the posterior PDF of model parameters is obtained from the prior PDF and the likelihood function using Bayes' Theorem given in Equation 14. The M-H algorithm is applied with a multivariate Gaussian for the proposal PDF, i.e. $q\left(\boldsymbol{\theta}^{\prime} \mid \boldsymbol{\theta}^{(\zeta)}\right)=\mathcal{N}\left(\boldsymbol{\theta}^{(\zeta)}, \boldsymbol{\Sigma}_{q}\right)$, where $\boldsymbol{\Sigma}_{q} \in \mathbb{R}^{d \times d}$ is the covariance matrix of the random walk. Given that model parameters are assumed to be stochastically independent a priori (recall Section 3.2), $\boldsymbol{\Sigma}_{q}$ is a diagonal matrix, i.e., $\boldsymbol{\Sigma}_{q}=\operatorname{diag}\left(\sigma_{q, 1}^{2}, \cdots, \sigma_{q, d}^{2}\right.$ ), and each individual parameter in $\boldsymbol{\theta}$ performs an independent random walk. The diagonal elements of $\boldsymbol{\Sigma}_{q}$ are appropriately selected through initial test runs such that the monitored acceptance rate (ratio between accepted M-H samples over total amount of samples) is within the suggested range $\bar{r} \in[0.2,0.4]$ for M-H algorithm [59]. See algorithm configuration in Table A.3.

As an example, the prior and updated PDFs of model parameters are presented in Figure A.4 for the most plausible model class $\mathcal{M}_{1}$, as shown further below. The mean and standard deviation of the updated parameters for model classes $\mathcal{M}_{1}$ to $\mathcal{M}_{5}$ are further summarized in Table A.4. As a comment, observe in Table A.4 that the estimated posterior mean of the bias parameter for the effective stiffness model $\mu_{e_{2}}$ takes non-zero values for all model classes. The corresponding posterior mean values for the bias parameter $\mu_{e_{1}}$ are also non-zero although they take relative lower values. It is interpreted as a systematic discrepancy the between model output and the data, which may be attributed to missing damage modes like delamination (see Figure A.2), among other causes. Thus, if a delamination model would have been considered within the equation of system response, it would probably have captured a part of such bias, leading to better inferences.

\subsection{Model class assessment}

According to the theory presented in Section 3.6, the choice for the most plausible model class among the set of candidate model classes is based on higher posterior probabilities, $p\left(\mathcal{M}_{j} \mid \mathcal{D}, \mathbf{M}\right)$. Results for model class assessment are shown in Table A.5 for classes $\mathcal{M}_{1}$ to $\mathcal{M}_{5}$ using a uniform prior $p\left(\mathcal{M}_{j} \mid \mathbf{M}\right)=1 / 5$ (i.e. all model classes are considered equally plausible a priori). Model class $\mathcal{M}_{1}$ (classical shear-lag) is revealed as the most evident to explain the observed damage data, hence the one that shows the best trade-off between datafit and model complexity, thus resulting in the highest posterior probability. In contrast, models classes $\mathcal{M}_{3}$ (interlaminar shear-lag) and $\mathcal{M}_{5}(\mathrm{COD})$, that involve more model parameters and more complex 
analysis, show negligible posterior probabilities through relatively low evidence values. A forward model simulation using $\mathcal{M}_{1}$ is shown in Figure A.5 in comparison with experimental damage data.

\section{Discussion of case study results}

The proposed Bayesian approach for model class selection is exemplified using the case study presented in the previous section. Several competing damage mechanics models were selected from the composites damage literature, which represent physically different models with different degrees of non-linearity and dimensionality. As apparent from the results, the model classes that involve more complex analysis (i.e., variational and COD analysis, $\mathcal{M}_{4}$ and $\mathcal{M}_{5}$ respectively) do not necessarily provide higher probabilities in explaining the observed damage response. This result contradicts the general conception that more complex analysis may be necessary to capture the various fatigue damage mechanisms and thus, to obtain better predictive performance. It is an example of the Principle of Model Parsimony in the context of fatigue damage modeling in composites, that comes into play through the Bayesian model selection approach by Equation 24. However, these results are based on data for one particular laminate configuration, so further investigations are needed to validate the results on other layups for a broader generalization.

More specifically, it can be observed in Table A.5 that the evidence reaches the lowest values for either models classes that involve more complex damage-mechanics analysis, like $\mathcal{M}_{5}$, or models classes that involve parameters which are difficult to establish priors for, like $\mathcal{M}_{3}$. These low values for the evidence can be explained based on the likelihood function, that is actually evaluated using prior samples from a region of the parameters space far from the region of high likelihood (recall Equation 23). It is a consequence of (1) using high-sensitivity parameters as model parameters within the model class, which favors a narrower concentration of the high-likelihood region over the parameter space, and (2) having diffuse prior information for some of those parameters (such as the normalized shear modulus $\frac{G}{d_{0}}$ in $\mathcal{M}_{3}$ ). This, in turn, enforces a larger distance between the prior and the posterior PDFs that leads to a larger EIG term, thereby penalizing the evidence by Equation 24 .

Notwithstanding, it should be noted that the use of high-sensitivity parameters does not automatically force the model class to extract more information from the data. It will ultimately depend on the "distance" between the likelihood function $p\left(\mathcal{D} \mid \boldsymbol{\theta}, \mathcal{M}_{j}\right)$ and the prior information $p\left(\boldsymbol{\theta} \mid \mathcal{M}_{j}\right)$ PDFs. In other words, the data make a difference only when they tell us something about the model parameters that the prior information does not [21]. This is typically the case for "fitting" parameters, that tend to capture much more information from data than, for example, mechanical parameters, as shown in Figure A.4. In this sense, the choice of widely dispersed priors for high-sensitivity parameters, such as non-informative priors, should be avoided since it leads to a huge information gain from the data. Thus, the contribution of the data-fit term (AGF) to the overall evidence of the model class may become negligible in relation to the high contribution 
of the EIG term, creating a bias in the model-class assessment problem [22]. Therefore, if such model class is utilized for future prediction, as arises in prognostics, the results are expected to significantly depend on the details of the data.

In summary, the results have highlighted the relevance of the information-theoretic approach for model class assessment in the context of fatigue damage modeling in composites. The amount of information that the model class needs to extract from the data to update its prior information emerges as a key variable for model class assessment. It actually determines the "information-theoretic complexity" of the model class, rather than the use of a more complex damage-mechanics approach or a larger number of parameters. Then, the evidence of the model class accounts for such information gain as a penalty term and implicitly enforces a quantitative Ockham's razor, such that simpler models that are consistent with data are favored through a healthy balance between the information gained from data and the average goodness of fit.

\section{Conclusion}

A Bayesian approach for quantifying modeling uncertainty is presented in application to several damage mechanics models in composites. A case study is presented using experimental fatigue damage data to illustrate the proposed methodology. The best class of models is chosen based on relative probability among all other candidate models. This probability is computed through Bayes' Theorem using the evidence for the model class given by data and the modeler's choice of prior probability for each model class. A key finding from this study is that the most simple shear-lag model turns out to be the most probable candidate when selected by striking a balance between average goodness of fit and amount of information extracted from data. More research effort is needed to incorporate the effects of other manifestations of damage such as delamination, and in general, to extend this approach to other laminate layups.

\section{Acknowledgements}

The two first authors would like to thank the Ministry of Education of Spain for the FPU grants AP20094641, AP2009-2390, the European Union for project GGI3000IDIB and the Prognostics Center of Excellence at NASA Ames Research Center, which kindly hosted them during the course of this work. They would also like to thank Prof. James L. Beck from California Institute of Technology for the valuable guidance through Bayesian methodology. Authors would also like to thank the Structures and Composites lab at Stanford University for experimental data and NASA ARMD/AvSafe project SSAT, which provided partial support for this work. 


\section{Appendix A. Nomenclature and basic relations}

For ply and laminate properties, the nomenclature exposed in Table A.6 is adopted in this work. Notice that subscripts $\{1,2,3\}$ refer to ply properties defined in local axis while subscripts $\{x, y, z\}$ refer to sub-laminate or laminate properties defined in global axis, that corresponds to the laminate coordinate system. The first local direction "1" coincides with fibers direction in a given ply or lamina, while directions "2-3" are the in-plane and out-of-plane transverse directions. For global axis, "x" refers to the fatigue loading direction, while "y-z" refers to the in-plane and out-of-plane transverse directions, respectively. In addition, the superscript $(\phi)$ denotes: "property referred to the $\left[\phi_{\frac{n_{\phi}}{2}}\right]$-sublaminate".

The function $a$ in Equation 1 is defined as a function of the laminate and ply properties listed in Table A.6, as follows:

$$
a=\frac{E_{2} t_{90}}{E_{1} t_{\phi}}\left(1-\nu_{x y}^{(\phi)} \frac{\frac{\nu_{x y}^{(\phi)} t_{90}}{E_{y}^{(\phi)}}+\frac{\nu_{12} t_{\phi}}{E_{2}}}{\frac{t_{90}}{E_{y}^{(\phi)}}+\frac{t_{\phi}}{E_{1}}}\right) \frac{1-\nu_{12} \nu_{x y}^{(\phi)}}{1-\nu_{12}^{2} \frac{E_{2}}{E_{1}}}
$$

The constants $\alpha_{1}, \alpha_{2}$ involved in Equation $2 \mathrm{~b}$ are defined as $\alpha_{1}=\frac{1}{2} \sqrt{2 \sqrt{q}-p}$ and $\alpha_{2}=\frac{1}{2} \sqrt{2 \sqrt{q}+p}$, where $p$ and $q$ are known functions of the ply properties defined as $p=\frac{C_{2}-C_{4}}{C_{3}}, q=\frac{C_{1}}{C_{3}}$. The terms $C_{i}, i$ : $\{1, \ldots, 4\}$ are calculated as follows:

$$
\begin{array}{r}
C_{1}=\frac{1}{E_{2}}+\frac{1}{\lambda E_{x}^{(\phi)}} \\
C_{2}=\left(\lambda+\frac{2}{3}\right) \frac{\nu_{23}}{E_{2}}-\frac{\lambda \nu_{x z}^{(\phi)}}{3 E_{x}^{(\phi)}} \\
C_{3}=(1+\lambda)\left(3 \lambda^{2}+12 \lambda+8\right) \frac{1}{60 E_{2}} \\
C_{4}=\frac{1}{3}\left(\frac{1}{G_{23}}+\frac{\lambda}{G_{x z}^{(\phi)}}\right)
\end{array}
$$

In the last equations, $\lambda=\frac{t_{\phi}}{t_{90}}$ and $\nu_{23}$ and $G_{23}$ are the out-of-plane Poisson ratio and shear modulus of the ply, respectively. Both can be related as $G_{23}=\frac{E_{2}}{2\left(1+\mu_{23}\right)}$. For cross-ply laminates, as the case study considered in Section $5, \phi=0^{\circ}$, and the laminate and sub-laminate global axis $\{x, y, z\}$ coincide with ply local axis $\{1,2,3\}$. In this particular case, the following identities hold:

$$
E_{x}^{(0)}=E_{1} ; \quad E_{y}^{(0)}=E_{2} ; \quad \nu_{x y}^{(0)}=\nu_{12} ; \quad G_{x y}^{(0)}=G_{12} ; \quad G_{x z}^{(0)}=G_{12}
$$

The rest of properties that appear in Equations A.1 to A.5 are defined in Table A.6. The initial longitudinal Young's modulus of the laminate $E_{x, 0}$ can be obtained using the classical laminate plate theory [45]; however, for the laminates considered in the case study it can be readily approximated using a simple rule of mixtures:

$$
E_{x, 0} \approx \frac{t_{0} E_{1}+t_{90} E_{2}}{t_{0}+t_{90}}
$$


Regarding the COD-based model [27], the matrices $\mathbf{A}^{k}$ are given by ( $k$ denotes that it is referred to the $k^{\text {th }}$ ply):

$$
\mathbf{A}^{k}=\mathbf{N}_{I}^{k}\left(\mathbf{S}^{k}\right)^{-1}
$$

where $\mathbf{S}^{k}$ is the in-plane compliance matrix of ply $k$ and $\mathbf{N}_{I}^{k}$ is a matrix defined by the vector normal to the surface of transverse crack, $\mathbf{n}^{k}$, as:

$$
\mathbf{N}_{I}^{k}=\left(\begin{array}{ccc}
n_{1}^{k} & 0 & n_{2}^{k} \\
0 & n_{2}^{k} & n_{1}^{k} \\
0 & 0 & 0
\end{array}\right), \mathbf{n}^{k}=\left(\begin{array}{c}
n_{1}^{k} \\
n_{2}^{k} \\
0
\end{array}\right)
$$

The matrix $\boldsymbol{\beta}^{k i}$ is related to the average crack opening displacement of matrix micro-cracks and also with the tension on the crack surface:

$$
\boldsymbol{\beta}^{k i}=0, \forall k \neq i ; \quad \boldsymbol{\beta}^{k k}=\left(\begin{array}{ccc}
\beta_{1}^{k} & 0 & 0 \\
0 & \beta_{2}^{k} & 0 \\
0 & 0 & \beta_{3}^{k}
\end{array}\right)
$$

where

$$
\begin{aligned}
& \beta_{1}^{k}=\frac{4}{\pi} \gamma_{1} \frac{\ln \left(\cosh \left(\frac{\pi t^{k} \rho^{k}}{2}\right)\right)}{\left(t^{k} \rho^{k}\right)^{2}} \\
& \beta_{2}^{k}=\frac{\pi}{2} \gamma_{2} \sum_{j=1}^{10} \frac{a_{j}}{\left(1+t^{k} \rho^{k}\right)^{j}} \\
& \beta_{3}^{k}=\frac{\pi}{2} \gamma_{3} \sum_{j=1}^{9} \frac{b_{j}}{\left(1+t^{k} \rho^{k}\right)^{j-2}} \\
& \gamma_{1}=\frac{1}{2 G_{12}} \\
& \gamma_{2}=\gamma_{3}=\frac{1}{E_{2}}-\frac{\nu_{12}^{2}}{E_{1}}
\end{aligned}
$$

The constants $a_{j}$ and $b_{j}$ can be found in the literature (see for example Table 1 in [27] or Table 4.2 in [23]). 


\section{References}

[1] K. Reifsnider, A. Talug, Analysis of fatigue damage in composite laminates, International Journal of Fatigue 2 (1) (1980) $3-11$.

[2] R. Jamison, K. Schulte, K. Reifsnider, W. Stinchcomb, Characterization and analysis of damage mechanisms in tensiontension fatigue of graphite/epoxy laminates, Effects of defects in composite materials, ASTM STP 836 (1984) 21-55.

[3] S. Sriramula, M. K. Chryssanthopoulos, Quantification of uncertainty modelling in stochastic analysis of FRP composites, Composites Part A: Applied Science and Manufacturing 40 (11) (2009) 1673-1684.

[4] C. Jiang, G. Liu, X. Han, A novel method for uncertainty inverse problems and application to material characterization of composites, Experimental Mechanics 48 (4) (2008) 539-548.

[5] S. Sakata, F. Ashida, T. Kojima, M. Zako, Influence of uncertainty in microscopic material property on homogenized elastic property of unidirectional fiber reinforced composites, Theoretical and Applied Mechanics Japan 56 (2008) $67-76$.

[6] P. Gayathri, K. Umesh, R. Ganguli, Effect of matrix cracking and material uncertainty on composite plates, Reliability Engineering \& System Safety 95 (7) (2010) 716-728.

[7] S. Sakata, F. Ashida, T. Kojima, M. Zako, Three-dimensional stochastic analysis using a perturbation-based homogenization method for elastic properties of composite material considering microscopic uncertainty, International Journal of Solids and Structures 45 (3-4) (2008) $894-907$.

[8] G. Georgiou, A. Manan, J. Cooper, Modeling composite wing aeroelastic behavior with uncertain damage severity and material properties, Mechanical Systems and Signal Processing 32 (2012) 32-43.

[9] C. Scarth, J. E. Cooper, P. M. Weaver, G. H. Silva, Uncertainty quantification of aeroelastic stability of composite plate wings using lamination parameters, Composite Structures 116 (0) (2014) 84 - 93.

[10] D. Lekou, T. Philippidis, Mechanical property variability in FRP laminates and its effect on failure prediction, Composites Part B: Engineering 39 (7) (2008) 1247-1256.

[11] M. Chiachío, J. Chiachío, G. Rus, Reliability in composites - A selective review and survey of current development, Composites Part B 43 (3) (2012) 902-913.

[12] R. Zhang, S. Mahadevan, Model uncertainty and Bayesian updating in reliability-based inspection, Structural Safety 22 (2) (2000) 145-160.

[13] R. Cross, A. Makeev, E. Armanios, Simultaneous uncertainty quantification of fracture mechanics based life prediction model parameters, International Journal of Fatigue 29 (8) (2007) 1510-1515.

[14] S. Sankararaman, Y. Ling, S. Mahadevan, Statistical inference of equivalent initial flaw size with complicated structural geometry and multi-axial variable amplitude loading, International Journal of Fatigue 32 (10) (2010) 1689-1700.

[15] X. Guan, R. Jha, Y. Liu, Model selection, updating, and averaging for probabilistic fatigue damage prognosis, Structural Safety 33 (3) (2011) 242-249.

[16] S. Sankararaman, Y. Ling, S. Mahadevan, Uncertainty quantification and model validation of fatigue crack growth prediction, Engineering Fracture Mechanics 78 (7) (2011) 1487-1504.

[17] S. Sankararaman, Y. Ling, C. Shantz, S. Mahadevan, Uncertainty quantification in fatigue crack growth prognosis, International Journal of Prognostics and Health Management 2 (1).

[18] X. Guan, Y. Liu, R. Jha, A. Saxena, J. Celaya, K. Geobel, Comparison of two probabilistic fatigue damage assessment approaches using prognostic performance metrics, International Journal of Prognostics and Health Management 2 (1).

[19] M. Gobbato, J. B. Kosmatka, J. P. Conte, A recursive Bayesian approach for fatigue damage prognosis: an experimental validation at the reliability component level, Mechanical Systems and Signal Processing 45 (2) (2014) $448-467$.

[20] M. Chiachío, J. Chiachío, G. Rus, J. L. Beck, Predicting fatigue damage in composites: A Bayesian framework, Structural Safety 51 (2014) $57-68$.

[21] E. Jaynes, Probability Theory: The Logic of Science, Cambridge University Press, 2003. 
[22] J. L. Beck, Bayesian system identification based on probability logic, Structural Control and Health Monitoring 17 (2010) $825-847$.

[23] R. Talreja, C. V. Singh, Damage and Failure of Composite Materials, Cambridge University Press, 2012.

[24] K. Garrett, J. Bailey, Multiple transverse fracture in $90^{\circ}$ cross-ply laminates of a glass fibre-reinforced polyester, Journal of Materials Science 12 (1) (1977) 157-168.

[25] A. Highsmith, K. Reifsnider, Stiffness-reduction mechanisms in composite laminates, Damage in composite materials, ASTM STP 775 (1982) 103-117.

[26] Z. Hashin, Analysis of cracked laminates: a variational approach, Mechanics of Materials 4 (2) (1985) 121-136.

[27] P. Gudmundson, Z. Weilin, An analytic model for thermoelastic properties of composite laminates containing transverse matrix cracks, International Journal of Solids and Structures 30 (23) (1993) 3211-3231.

[28] P. Lundmark, J. Varna, Constitutive relationships for laminates with ply cracks in in-plane loading, International Journal of Damage Mechanics 14 (3) (2005) 235-259.

[29] J. Nairn, S. Hu, The initiation and growth of delaminations induced by matrix microcracks in laminated composites, International Journal of Fracture 57 (1) (1992) 1-24.

[30] A. Saltelli, M. Ratto, T. Andres, F. Campolongo, J. Cariboni, D. Gatelli, M. Saisana, S. Tarantola, Global Sensitivity Analysis: The Primer, Wiley-Interscience, 2008.

[31] A. Saxena, K. Goebel, C. Larrosa, F. Chang, CFRP Composites dataset, NASA Ames Prognostics Data Repository, [http://ti.arc.nasa.gov/project/prognostic-data-repository], NASA Ames, Moffett Field, CA (2008).

[32] A. Saxena, K. Goebel, C. Larrosa, V. Janapati, S. Roy, F. Chang, Accelerated aging experiments for prognostics of damage growth in composites materials, in: The 8th International Workshop on Structural Health Monitoring, F.-K. Chang, Editor., Vol. 15, 2011.

[33] J. A. Nairn, in: R. Talreja, M. J.A.E. (Eds.), Polymer Matrix Composites, Elsevier Science, Amsterdam, 2000, Ch. Matrix Microcracking in Composites, pp. 403-432.

[34] J. Lee, D. Allen, C. Harris, Internal state variable approach for predicting stiffness reductions in fibrous laminated composites with matrix cracks, Journal of Composite Materials 23 (12) (1989) 1273-1291.

[35] P. Beaumont, R. Dimant, H. Shercliff, Failure processes in composite materials: getting physical, Journal of Materials Science 41 (20) (2006) 6526-6546.

[36] J. Reddy, Energy Principles and Variational Methods in Applied Mechanics, Wiley, 2002.

[37] C. Dym, I. H. Shames, Solid Mechanics: A Variational Approach, Augmented Edition, SpringerLink: Bücher, Springer, 2013.

[38] J. Varna, N. V. Akshantala, R. Talreja, Crack opening displacement and the associated response of laminates with varying constraints, International Journal of Damage Mechanics 8 (2) (1999) 174-193.

[39] R. Joffe, A. Krasnikovs, J. Varna, Cod-based simulation of transverse cracking and stiffness reduction in $\left[\mathrm{S} / 90_{n}\right]_{s}$ laminates, Composites Science and Technology 61 (5) (2001) 637-656.

[40] R. Joffe, J. Varna, Analytical modeling of stiffness reduction in symmetric and balanced laminates due to cracks in 90 layers, Composites Science and Technology 59 (11) (1999) 1641-1652.

[41] P. W. Manders, T.-W. Chou, F. R. Jones, J. W. Rock, Statistical analysis of multiple fracture in 0/90/0 glass fibre/epoxy resin laminates, Journal of Materials Science 18 (10) (1983) 2876-2889.

[42] R. Nuismer, S. Tan, Constitutive relations of a cracked composite lamina, Journal of Composite Materials 22 (4) (1988) $306-321$.

[43] S. Tan, R. Nuismer, A theory for progressive matrix cracking in composite laminates, Journal of Composite Materials 23 (10) (1989) 1029-1047.

[44] J. Nairn, S. Hu, in: R. Talreja (Ed.), Damage Mechanics of Composites Materials, Elsevier, 1994, Ch. Micromechanics of 
damage: A case study of matrix microcracking, pp. 187-214.

[45] J. Reddy, Mechanics of Laminated Composite Plates and Shells: Theory and Analysis, Second Edition, CRC Mechanical Engineering Series.

[46] J. A. Nairn, The strain energy release rate of composite microcracking: a variational approach, Journal of Composite Materials 23 (11) (1989) 1106-1129.

[47] J. A. Nairn, Some new variational mechanics results on composite microcracking, in: Proc. 10th International Conference on Composite Materials (ICCM-10) Whistler BC, Canada, 1995.

[48] E. T. Jaynes, Papers on Probability, Statistics and Statistical physics, (Ed. R.D. Rosenkrantz) Kluwer Academic Publishers, 1983.

[49] A. Saltelli, M. Ratto, S. Tarantola, F. Campolongo, Sensitivity analysis practices: Strategies for model-based inference, Reliability Engineering \& System Safety 91 (10) (2006) 1109-1125.

[50] E. Jaynes, Information theory and statistical mechanics, The Physical Review 106 (4) (1957) 620-630.

[51] J. L. Beck, K. V. Yuen, Model selection using response measurements: Bayesian probabilistic approach, Journal of Engineering Mechanics 130 (2004) 192.

[52] S. H. Cheung, J. L. Beck, Calculation of posterior probabilities for bayesian model class assessment and averaging from posterior samples based on dynamic system data, Computer-Aided Civil and Infrastructure Engineering 25 (5) (2010) 304-321.

[53] M. Muto, J. L. Beck, Bayesian updating and model class selection for hysteretic structural models using stochastic simulation, Journal of Vibration and Control 14 (1-2) (2008) 7-34.

[54] S. Kullback, R. A. Leibler, On information and sufficiency, The Annals of Mathematical Statistics 22 (1) (1951) 79-86.

[55] F. Liang, C. Liu, J. Chuanhai, Advanced Markov Chain Monte Carlo Methods, Wiley Online Library, 2010.

[56] N. Metropolis, A. W. Rosenbluth, M. N. Rosenbluth, A. H. Teller, E. Teller, Equation of state calculations by fast computing machines, The Journal of Chemical Physics 21 (1953) 1087-1092.

[57] W. Hastings, Monte Carlo sampling methods using Markov chains and their applications, Biometrika 57 (1) (1970) 97-109.

[58] C. Larrosa, F. Chang, Real time in-situ damage classification, quantification and diagnosis for composite structures, in: Proceedings of the 19th International Congress on Sound and Vibration, Vol. 15, 2012.

[59] G. Roberts, J. Rosenthal, Optimal scaling for various Metropolis-Hastings algorithms, Statistical Science 16 (4) (2001) $351-367$ 


\section{List of Figures}

A.1 Implementation scheme of M-H algorithm using the GPU to accelerate the computation of likelihood function. Note that M-H runs serially on the CPU, while the GPU executes part of the likelihood in parallel. . . . . . . . . . . . . . . . . .

A.2 Fatigue experiment for a T700G CFRP $\left[0_{2} / 90_{4}\right]_{\mathrm{S}}$ laminate. Shown in the left is the in situ set-up of the specimen on the testing machine. Observe the SHM system based on PZT sensors (SMART LayerR from Acellent Technologies Inc), placed on top and bottom of the specimen. The right panel shows a X-ray image of the specimen after 100 fatigue cycles. The bright white areas denote delaminated interfaces whereas the horizontal white lines are matrix

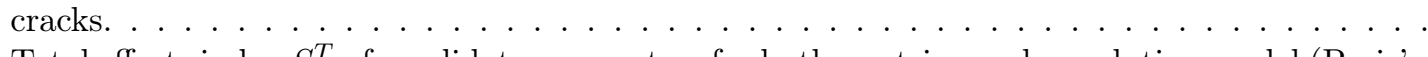

A.3 Total effects index $S_{i}^{T}$ of candidate parameters for both, matrix-cracks evolution model (Paris' law, Eq. 7) and effective stiffness model (Eqs. 1 to 4), using the approaches: (a) \& (d) classical shear-lag, (b) \& (e) bi-dimensional shear-lag, (c) \& (f) interlaminar shear-lag, (g) \& (i) variational, (h) \& (j) COD. Observe that the ply properties $E_{1}, E_{2}$ and $t$ are revealed as influential parameters in all model classes. . . . . . . . . . . . . . . . . . . . .

A.4 Normalized histograms for the marginalized posterior PDFs $p\left(\theta_{i} \mid \mathcal{D}, \mathcal{M}_{1}\right), i=1 \ldots 8$, after updating model class $\mathcal{M}_{1}$ (classical shear-lag). The posterior mean estimate for each parameter is represented by the vertical-dashed line. Note that the "difference" between the prior and the posterior PDF is larger for fitting parameter $\alpha$ than for mechanical parameters $E_{1}, E_{2}, t$.

A.5 Simulated damage response using the posterior PDF of parameters for model class $\mathcal{M}_{1}($ classical shear-lag). Observe that bounds cover well the experimental realizations. . . . . . . . . . 27 


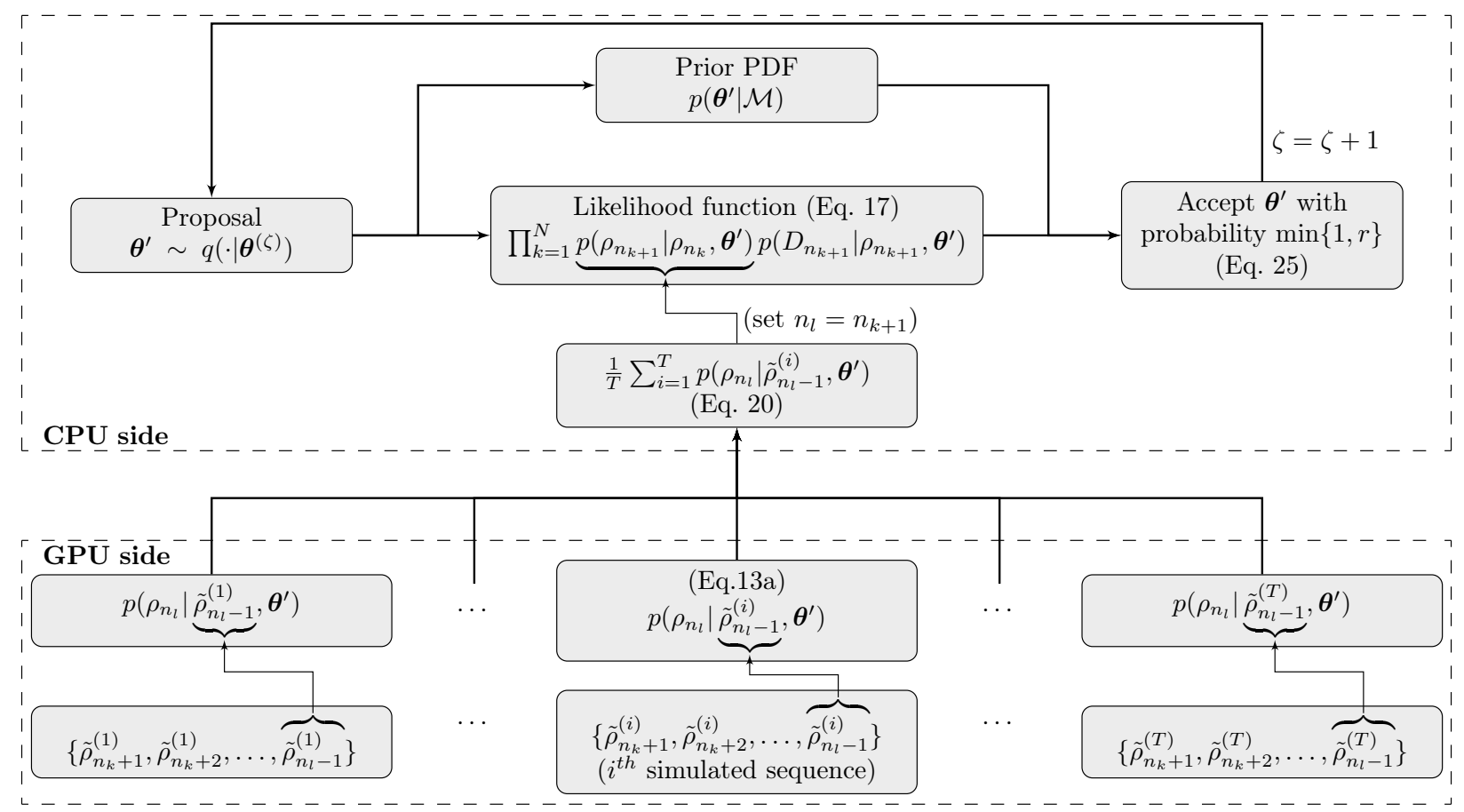

Figure A.1

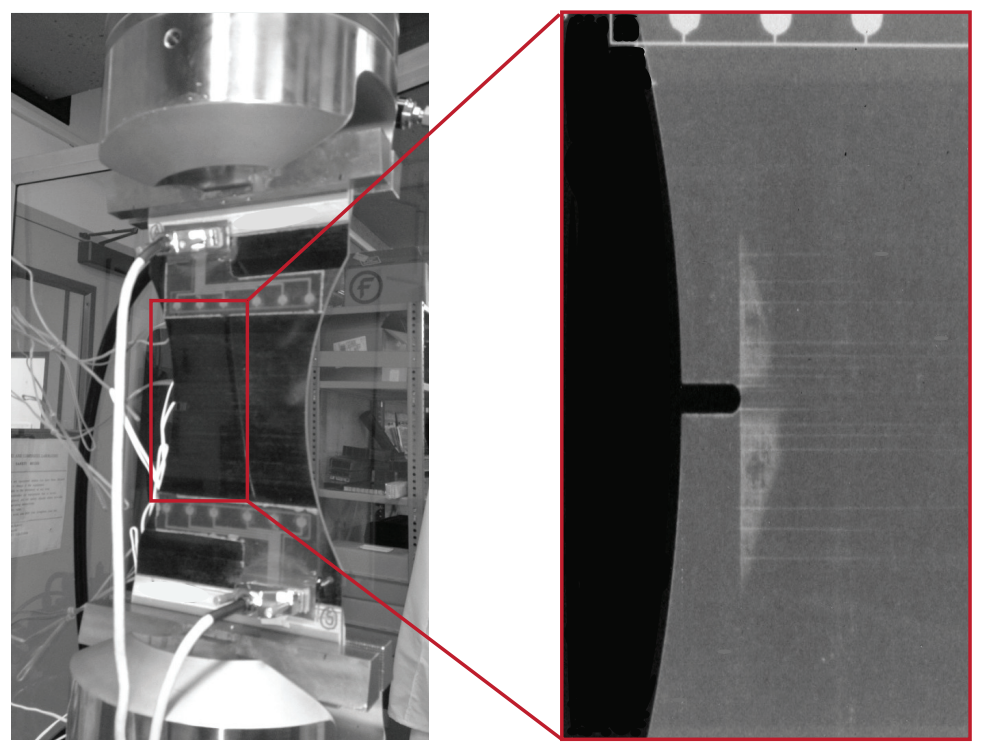

Figure A.2 


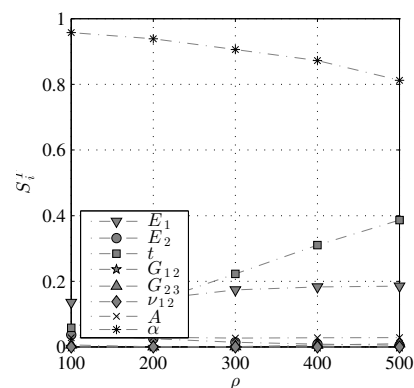

(a)

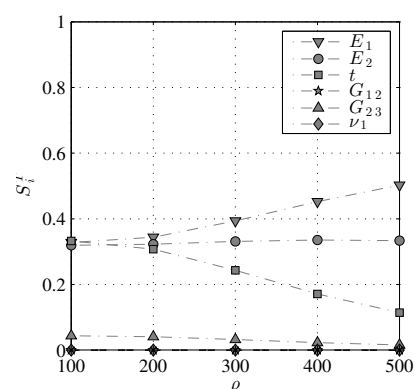

(d)

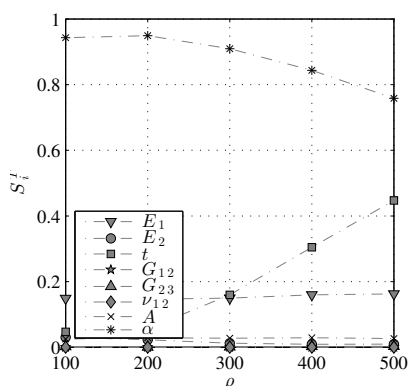

(g)

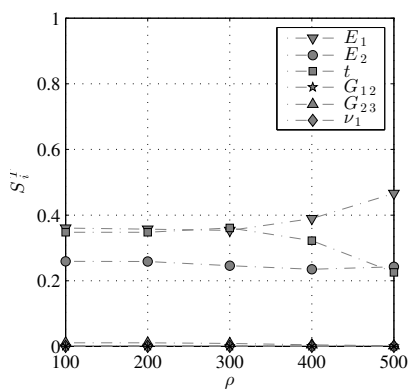

(i)

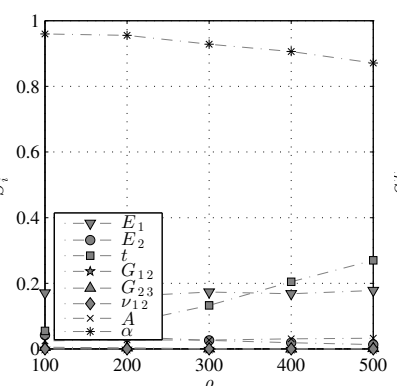

(b)

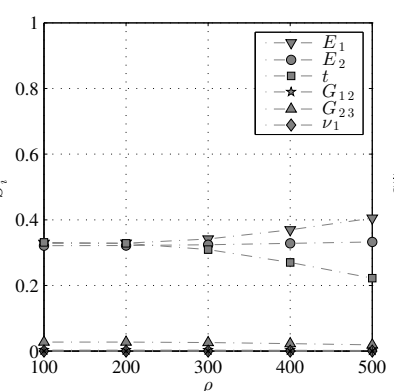

(e)

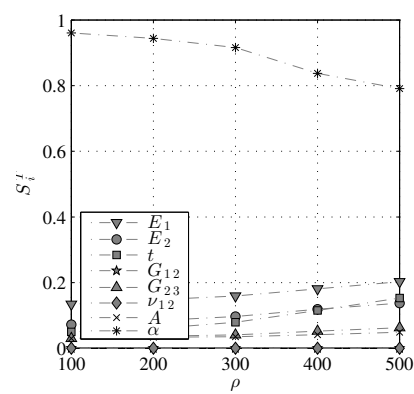

(h)

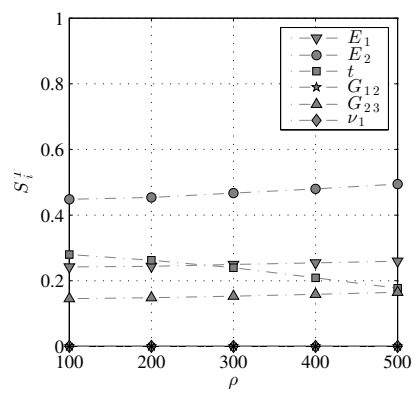

(j)

Figure A.3 


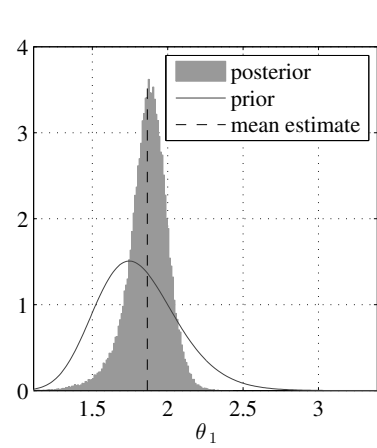

(a)

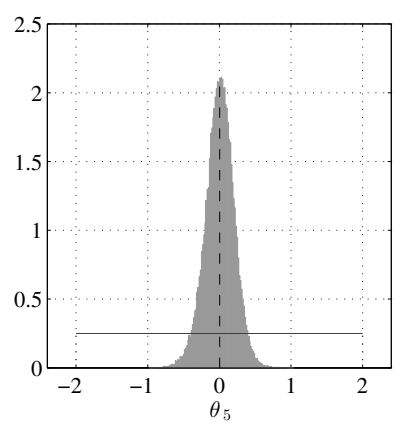

(e)

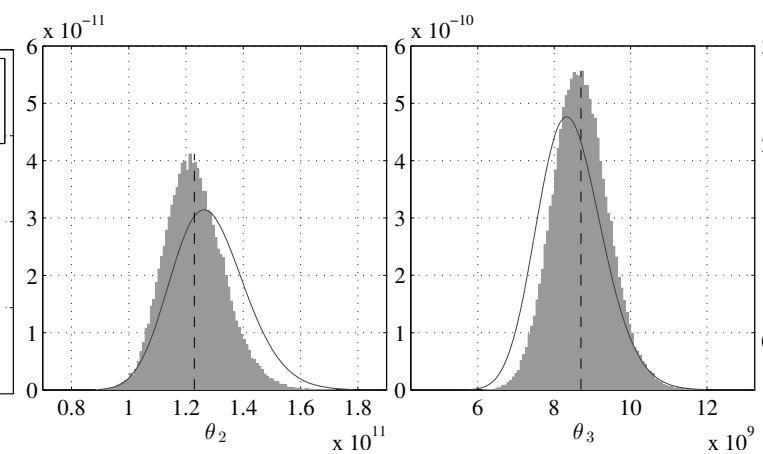

(b)

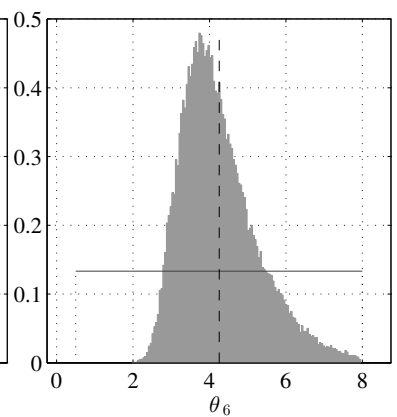

(f)

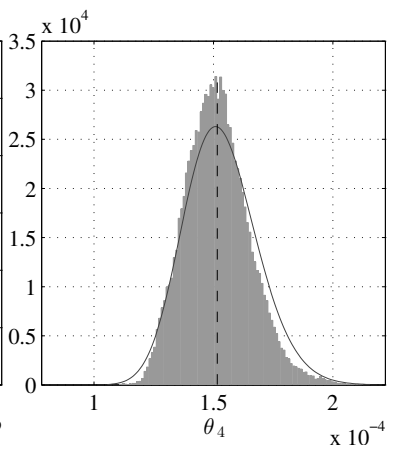

(d)

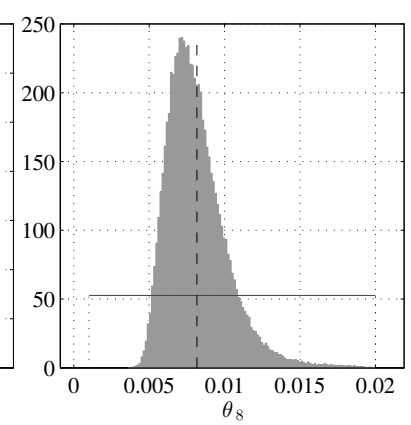

(h)

Figure A.4

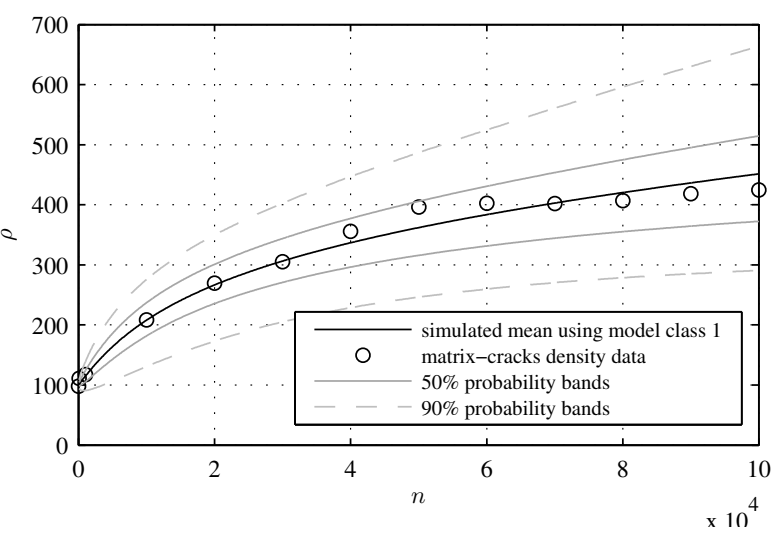

(a)

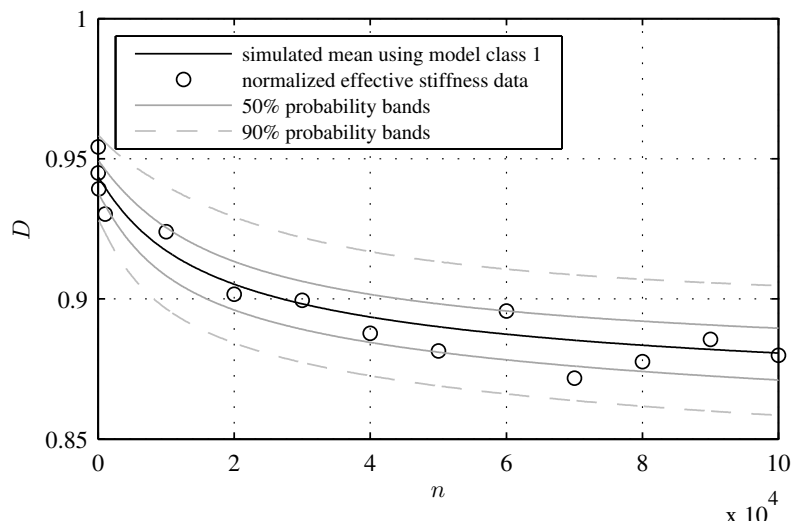

(b)

Figure A.5 


\begin{tabular}{|c|c|c|c|c|c|c|c|c|c|c|c|c|c|}
\hline Fatigue cycles, $n$ & $10^{1}$ & $10^{2}$ & $10^{3}$ & $10^{4}$ & $2 \cdot 10^{4}$ & $3 \cdot 10^{4}$ & $4 \cdot 10^{4}$ & $5 \cdot 10^{4}$ & $6 \cdot 10^{4}$ & $7 \cdot 10^{4}$ & $8 \cdot 10^{4}$ & $9 \cdot 10^{4}$ & $10^{5}$ \\
\hline$\rho_{n}[\#$ cracks $/ m]$ & 98.2 & 111.0 & 117.4 & 208.5 & 269.6 & 305.0 & 355.5 & 396.4 & 402.3 & 402.1 & 407.0 & 418.5 & 424.5 \\
\hline$D_{n}$ & 0.954 & 0.939 & 0.930 & 0.924 & 0.902 & 0.899 & 0.888 & 0.881 & 0.896 & 0.872 & 0.877 & 0.885 & 0.880 \\
\hline
\end{tabular}

Table A.1: Experimental sequence of damage for the cross-ply $\left[0_{2} / 90_{4}\right]_{\mathrm{s}}$ Torayca T700 CFRP laminate used in the case study. The data are presented for micro-cracks density $\left(\rho_{n}\right)$ and normalized effective stiffness $\left(D_{n}\right)$.

\begin{tabular}{|c|c|c|c|c|}
\hline Type & Parameter & Nominal value & Units & Prior PDF \\
\hline \multirow[t]{7}{*}{ Mechanical } & $E_{1}$ & $127.55 \cdot 10^{9}$ & $\mathrm{~Pa}$ & $\mathcal{L} \mathcal{N}\left(\ln \left(127.55 \cdot 10^{9}\right), 0.1\right)$ \\
\hline & $E_{2}$ & $8.41 \cdot 10^{9}$ & $\mathrm{~Pa}$ & $\mathcal{L N}\left(\ln \left(8.41 \cdot 10^{9}\right), 0.1\right)$ \\
\hline & $G_{12}$ & $6.20 \cdot 10^{9}$ & $\mathrm{~Pa}$ & $\mathcal{L N}\left(\ln \left(6.20 \cdot 10^{9}\right), 0.1\right)$ \\
\hline & $\frac{G_{m}}{d_{0}}$ & $1 \cdot 10^{14}$ & $\mathrm{~Pa} / m$ & $\mathcal{L N}\left(\ln \left(1 \cdot 10^{14}\right), 0.5\right)$ \\
\hline & $\nu_{12}$ & 0.31 & - & $\mathcal{L} \mathcal{N}(\ln (0.31), 0.1)$ \\
\hline & $G_{23}$ & $2.82 \cdot 10^{9}$ & $\mathrm{~Pa}$ & $\mathcal{L N}\left(\ln \left(2.82 \cdot 10^{9}\right), 0.1\right)$ \\
\hline & $t$ & $1.5 \cdot 10^{-4}$ & $m$ & $\mathcal{L N}\left(\ln \left(1.5 \cdot 10^{-4}\right), 0.1\right)$ \\
\hline \multirow[t]{2}{*}{ Fitting } & $\alpha$ & 1.80 & - & $\mathcal{L N}(\ln (1.80), 0.2)$ \\
\hline & $A$ & $1 \cdot 10^{-4}$ & - & $\mathcal{L N}\left(\ln \left(1 \cdot 10^{-4}\right), 0.2\right)$ \\
\hline \multirow[t]{4}{*}{ Errors } & $\mu_{e_{1}}$ & Not applicable & $\frac{\# \text { cracks }}{m \cdot \text { cycle }}$ & $\mathcal{U}(-2,2)$ \\
\hline & $\sigma_{e_{1}}$ & Not applicable & $\frac{\# \cdot \text { cracks }}{m \cdot \text { cycle }}$ & $\mathcal{U}(0.5,8)$ \\
\hline & $\mu_{e_{2}}$ & Not applicable & - & $\mathcal{U}(-0.08,0)$ \\
\hline & $\sigma_{e_{2}}$ & Not applicable & - & $\mathcal{U}(0.001,0.02)$ \\
\hline
\end{tabular}

Table A.2: Prior information of parameters used in calculations. The rest of parameters in damage mechanics models (Eq. 1 to 4) are obtained using the classical laminate plate theory [45] and the relations given in Appendix A.

The nominal values for fitting parameters have been defined through initial fitting tests.

\begin{tabular}{lccccr}
\hline & $\mathcal{M}_{1}$ & $\mathcal{M}_{2}$ & $\mathcal{M}_{3}$ & $\mathcal{M}_{4}$ & $\mathcal{M}_{5}$ \\
\hline$\sigma_{q, i}$ (Prop. std. dev.) & $4 \%$ & $4 \%$ & $2 \%$ & $3.5 \%$ & $1.5 \%$ \\
$N_{s}$ (M-H samples) & $5 \cdot 10^{5}$ & $5 \cdot 10^{5}$ & $10^{6}$ & $5 \cdot 10^{5}$ & $10^{6}$ \\
$T$ (Eq. 19) & $10^{4}$ & $10^{4}$ & $10^{4}$ & $10^{4}$ & $10^{4}$ \\
\hline
\end{tabular}

Table A.3: Metropolis Hastings algorithm configuration for models classes $\mathcal{M}_{1}$ to $\mathcal{M}_{5}$. The diagonal elements $\sigma_{q, i}$ of covariance matrix $\boldsymbol{\Sigma}_{q}$ are defined as the specified percent (1st row) of the $5^{t h}-95^{t h}$ inter-percentile range of the prior PDFs for each of the $i^{\text {th }}$ component of the parameter vector, $i=1, \ldots, d$. 


\begin{tabular}{|c|c|c|c|c|c|c|c|c|c|c|c|}
\hline & & \multirow{2}{*}{$\begin{array}{l}\text { Fitting param. } \\
\qquad \alpha\end{array}$} & \multicolumn{5}{|c|}{ Mechanical parameters } & \multicolumn{4}{|c|}{ Error param. } \\
\hline & & & $\begin{array}{c}E_{1} \\
\left(\times 10^{9}\right) \\
\end{array}$ & $\begin{array}{c}E_{2} \\
\left(\times 10^{9}\right) \\
\end{array}$ & $\begin{array}{c}t \\
\left(\times 10^{-4}\right)\end{array}$ & $\begin{array}{c}\frac{G_{m}}{d_{0}} \\
\left(\times 10^{14}\right)\end{array}$ & $\begin{array}{c}G_{23} \\
\left(\times 10^{9}\right) \\
\end{array}$ & $\begin{array}{c}\mu_{e_{1}} \\
\left(\times 10^{-1}\right)\end{array}$ & $\sigma_{e_{1}}$ & $\begin{array}{c}\mu_{e_{2}} \\
\left(\times 10^{-2}\right)\end{array}$ & $\begin{array}{r}\sigma_{e_{2}} \\
\left(\times 10^{-3}\right) \\
\end{array}$ \\
\hline $\mathcal{M}_{1}$ & $\begin{array}{l}\text { mean } \\
\text { std }\end{array}$ & $\begin{array}{l}1.86 \\
0.13\end{array}$ & $\begin{array}{c}123.16 \\
10.3\end{array}$ & $\begin{array}{l}8.72 \\
0.73\end{array}$ & $\begin{array}{l}1.52 \\
0.14\end{array}$ & $\begin{array}{l}- \\
-\end{array}$ & $\begin{array}{l}- \\
-\end{array}$ & $\begin{array}{l}0.05 \\
2.02\end{array}$ & $\begin{array}{l}4.25 \\
0.99\end{array}$ & $\begin{array}{c}-2.92 \\
0.64\end{array}$ & $\begin{array}{l}8.1 \\
1.9\end{array}$ \\
\hline $\mathcal{M}_{2}$ & $\begin{array}{l}\text { mean } \\
\text { std }\end{array}$ & $\begin{array}{l}1.96 \\
0.15\end{array}$ & $\begin{array}{c}116.21 \\
10.2\end{array}$ & $\begin{array}{l}9.28 \\
0.81\end{array}$ & $\begin{array}{l}1.61 \\
0.14\end{array}$ & $\begin{array}{l}- \\
-\end{array}$ & $\begin{array}{l}- \\
-\end{array}$ & $\begin{array}{c}-2.35 \\
3.22\end{array}$ & $\begin{array}{l}4.27 \\
1.00\end{array}$ & $\begin{array}{c}-3.95 \\
0.64\end{array}$ & $\begin{array}{l}8.7 \\
2.2\end{array}$ \\
\hline $\mathcal{M}_{3}$ & $\begin{array}{l}\text { mean } \\
\text { std }\end{array}$ & $\begin{array}{l}2.03 \\
0.21\end{array}$ & $\begin{array}{c}134.43 \\
10.9\end{array}$ & $\begin{array}{l}9.98 \\
0.96\end{array}$ & $\begin{array}{l}1.59 \\
0.15\end{array}$ & $\begin{array}{l}0.25 \\
0.13\end{array}$ & $\begin{array}{l}- \\
- \\
-\end{array}$ & $\begin{array}{c}-6.37 \\
6.89\end{array}$ & $\begin{array}{l}4.41 \\
1.03\end{array}$ & $\begin{array}{l}-4.9 \\
1.01\end{array}$ & $\begin{array}{r}10.4 \\
3.1\end{array}$ \\
\hline $\mathcal{M}_{4}$ & $\begin{array}{l}\text { mean } \\
\text { std }\end{array}$ & $\begin{array}{l}1.89 \\
0.12\end{array}$ & $\begin{array}{c}124.54 \\
10.4\end{array}$ & $\begin{array}{l}8.59 \\
0.75\end{array}$ & $\begin{array}{l}1.54 \\
0.14\end{array}$ & $\begin{array}{l}- \\
- \\
-\end{array}$ & $\begin{array}{l}- \\
- \\
-\end{array}$ & $\begin{array}{c}-1.52 \\
2.86\end{array}$ & $\begin{array}{l}3.95 \\
0.68\end{array}$ & $\begin{array}{c}-3.55 \\
0.58\end{array}$ & $\begin{array}{l}8.4 \\
2.1\end{array}$ \\
\hline $\mathcal{M}_{5}$ & $\begin{array}{l}\text { mean } \\
\text { std }\end{array}$ & $\begin{array}{l}1.99 \\
0.15\end{array}$ & $\begin{array}{c}133.21 \\
12.3\end{array}$ & $\begin{array}{l}7.60 \\
0.52\end{array}$ & $\begin{array}{l}1.46 \\
0.14\end{array}$ & $\begin{array}{l}- \\
- \\
-\end{array}$ & $\begin{array}{l}2.99 \\
0.28\end{array}$ & $\begin{array}{c}-13.6 \\
7.93\end{array}$ & $\begin{array}{l}4.30 \\
0.98\end{array}$ & $\begin{array}{c}-3.08 \\
0.62\end{array}$ & $\begin{array}{l}8.3 \\
1.9\end{array}$ \\
\hline
\end{tabular}

Table A.4: Mean and standard deviation of the updated model parameters for models classes $\mathcal{M}_{1}$ to $\mathcal{M}_{5}$, estimated from samples of the marginal posterior PDFs. Units are specified in Table A.2. In this table, the set of parameters that compound each model class is also found, e.g., for $\mathcal{M}_{5}, \boldsymbol{\theta}=\left\{\alpha, E_{1}, E_{2}, t, G_{23}, \mu_{e_{1}}, \sigma_{e_{1}}, \mu_{e_{2}}, \sigma_{e_{2}}\right\}$

\begin{tabular}{lcccc}
\hline Model class & Log evidence & EIG & AGF & Posterior Probability \\
\hline $\mathcal{M}_{1}$ (classical SL) & -9.99 & 3.73 & -6.26 & $\mathbf{0 . 7 4 5}$ \\
$\mathcal{M}_{2}$ (2D-SL) & -10.80 & 4.22 & -6.58 & 0.115 \\
$\mathcal{M}_{3}$ (interlaminar SL) & -16.62 & $\mathbf{8 . 8 8}$ & -7.74 & $1.75 \cdot 10^{-7}$ \\
$\mathcal{M}_{4}$ (variational) & -10.72 & 4.47 & -6.25 & 0.139 \\
$\mathcal{M}_{5}$ (COD) & -14.16 & 7.95 & $\mathbf{- 6 . 2 1}$ & $5.02 \cdot 10^{-5}$ \\
\hline
\end{tabular}

Table A.5: Results of Bayesian model class assessment. The 2nd column (Log evidence) is the difference of the next two columns $(\mathrm{EIG}=$ Expected Information Gain, $\mathrm{AGF}=$ Average Goodness of Fit $)$. The 5th column is the estimated posterior probability of each model class, i.e., $p\left(\mathcal{M}_{j} \mid \mathcal{D}, \mathbf{M}\right)$, using a uniform prior $p\left(\mathcal{M}_{j} \mid \mathbf{M}\right)=1 / 5$. Note that $\mathcal{M}_{5}$ provides a negligible posterior probability even though it reaches the best average goodness of fit.

\begin{tabular}{|c|c|c|c|c|c|}
\hline \multirow[t]{4}{*}{ Laminate } & $E_{x, 0}$ & Initial longitudinal Young's modulus & & $t_{\phi}$ & {$\left[\phi_{\frac{n_{\phi}}{2}}\right]$-sublaminate thickness } \\
\hline & $E_{x}^{*}$ & Effective long. Young's modulus & Ply & $t$ & Ply thickness \\
\hline & $h^{x}$ & Laminate half-thickness & & $d_{0}$ & Interlaminar layer thickness \\
\hline & $B$ & Laminate half-width & & $G_{m}$ & Interlaminar layer shear modulus \\
\hline \multirow[t]{6}{*}{ Sublaminate } & $E_{x}^{(\phi)}$ & Longitudinal Young's modulus & & $E_{1}$ & Longitudinal Young's modulus \\
\hline & $E_{y}^{(\phi)}$ & Transverse Young's modulus & & $E_{2}$ & Transverse Young's modulus \\
\hline & $\nu_{x y}^{(\phi)}$ & In-plane Poisson ratio & & $\nu_{12}$ & In-plane Poisson ratio \\
\hline & $G_{x y}^{(\phi)}$ & In-plane shear modulus & & $\nu_{23}$ & Out-of-plane Poisson ratio \\
\hline & $G_{x z}^{(\phi)}$ & Out-of-plane shear modulus & & $G_{12}$ & In-plane shear modulus \\
\hline & $t_{90}$ & {$\left[90_{n_{90}}\right]$-sublaminate half-thickness } & & $G_{23}$ & Out-of-plane shear modulus \\
\hline
\end{tabular}

Table A.6: Nomenclature table. Nominal values of main ply and geometry parameters are provided in Table A.2 